\title{
A Vertex-Centered and Positivity-Preserving Finite Volume Scheme for Two-Dimensional Three-Temperature Radiation Diffusion Equations on General Polygonal Meshes
}

\author{
Shuai $\mathrm{Su}^{1}$ and Jiming $\mathrm{Wu}^{2, *}$ \\ ${ }^{1}$ Graduate School of China Academy of Engineering Physics, Beijing 100088, \\ China \\ ${ }^{2}$ Institute of Applied Physics and Computational Mathematics, P. O. Box 8009, \\ Beijing 100088, China
}

Received 28 November 2018; Accepted (in revised version) 21 May 2019

\begin{abstract}
Two-dimensional three-temperature (2-D 3-T) radiation diffusion equations are widely used to approximately describe the evolution of radiation energy within a multimaterial system and explain the exchange of energy among electrons, ions and photons. In this paper, we suggest a new positivity-preserving finite volume scheme for 2-D 3-T radiation diffusion equations on general polygonal meshes. The vertex unknowns are treated as primary ones for which the finite volume equations are constructed. The edge-midpoint and cell-centered unknowns are used as auxiliary ones and interpolated by the primary unknowns, which makes the final scheme a pure vertex-centered one. By comparison, most existing positivity-preserving finite volume schemes are cell-centered and based on the convex decomposition of the co-normal. Here, the co-normal decomposition is not convex in general, leading to a fixed stencil of the flux approximation and avoiding a certain search algorithm on complex grids. Moreover, the new scheme effectively alleviates the numerical heat-barrier issue suffered by most existing cell-centered or hybrid schemes in solving strongly nonlinear radiation diffusion equations. Numerical experiments demonstrate the second-order accuracy and the positivity of the solution on various distorted grids. For the problem without analytic solution, the contours of the numerical solutions obtained by our scheme on distorted meshes accord with those on smooth quadrilateral meshes.
\end{abstract}

AMS subject classifications: 65M08, 65M22

Key words: 2-D 3-T, radiation diffusion equations, vertex-centered scheme, positivitypreserving, finite volume.

\footnotetext{
${ }^{*}$ Corresponding author. Email addresses: sushuai16@gscaep.ac.cn (S. Su), wu_jiming@iapcm.ac.cn (J. M. Wu) 


\section{Introduction}

Radiation diffusion equations arise in a wide range of applications such as radiation hydrodynamics and astrophysical plasmas. If the radiation field is not in thermodynamic equilibrium with the material, a system of coupled time-dependent diffusion equations, which is called non-equilibrium radiation diffusion equations, is used to describe the energy transport. Due to its strong nonlinear phenomena, strong discontinuous interfaces and tight coupling, the numerical simulation of such equations is very challenging and has drawn many researchers' attention.

In recent years, some efficient numerical methods were suggested for solving the non-equilibrium two dimensional two-temperature (2-D 2-T) radiation diffusion equations $[5,15-17,28-30,32,46,48]$. Most of them focused on high-order time integrations and the nonlinear iteration technique on rectangular meshes. Meanwhile, some monotone schemes were also studied. In [51], the authors designed two finite volume element schemes and proved that one of them is monotonic under some geometric conditions and another is monotonic under some repairing techniques. In [12], a monotone tailored finite point method was suggested for solving the non-equilibrium radiation diffusion equations. Recently, a moving mesh finite difference method was proposed in [44].

In the simulation of laser-driven implosion of a fuel capsule in inertial confinement fusion experiments, a finer and more complicated model, involving the twodimensional three-temperature (2-D 3-T) radiation diffusion equations, is widely used to describe the evolution and exchange of energy among electrons, ions and photons $[6,11,14,18,27]$. Since 2000 s, some numerical methods have been developed for 2-D 3-T radiation equations, such as finite volume method and finite element method. In [27], a fully implicit finite volume scheme combined with parallel adaptive multigrid method was suggested in the framework of UG. In [14], the authors designed a symmetric finite volume element (SFVE) method with a presconditioning technique, a mesh adaptation algorithm and a two-grid procedure. A two-level iterative method was proposed in [43] for the numerical simulation of 2-D 3-T radiation diffusion equations, based on the Jacobian-free Newton-Krylov (JFNK) framework for preconditioning. In [47], two substructuring nonoverlapping domain decomposition preconditioners were employed to solve the SFVE discretization of 2-D 3-T radiation diffusion equations with strongly discontinuous coefficients. In [7], two finite volume element schemes were constructed on triangular meshes. The authors in [31] adopted the freezing coefficient method to linearize the nonlinear equations and then solved the resulting equations by Raviart-Thomas mixed finite element method.

Unfortunately, numerical methods mentioned above for 2-D 3-T radiation diffusion equations were discussed only on triangular or quadrilateral meshes. In many applications such as radiation hydrodynamics (RHD), the meshes are typically distorted, concave or have hanging nodes due to the complex fluid flow. Hence, solving 3-T radiation diffusion equations on distorted polygonal meshes is very interesting and important. In [45], a Lions domain decomposition algorithm based on a cell functional 
minimization scheme was studied on non-matching multi-block grids for nonlinear radiation diffusion equations. In [4], interface reconstruction was implemented within any cell that has more than one material and a new numerical scheme was developed for the 3-T radiation diffusion equations on polygonal or polyhedral meshes. The authors in [37] used a monotone cell-centered scheme for diffusion equation to discrete the 3-T radiation diffusion equations on convex polygons and adopted the interpolation algorithms suggested in [8] to evaluate the vertex unknowns. To design a discrete scheme for 2-D 3-T diffusion equations on arbitrary polygonal meshes is one of the main motivations here.

In many situations, another significant requirement of the numerical schemes for 2-D 3-T radiation diffusion equations is that the discrete solution should be nonnegative. However, numerical schemes mentioned in the previous paragraph do not possess the positivity-preserving property unconditionally. The first scheme in [7] can preserve the positivity of the solutions if and only if the maximal interior angle of the primary partition is not greater than $\pi / 2$, while the second scheme adopted two post-processing techniques, i.e., global repair technique $[22,41]$ and cutoff method [21], to keep the positivity-preserving property, which may possibly destroy the accuracy and the energy conservation. Hence, for 2-D 3-T equations on general polygonal meshes, it is interesting to design a strictly positivity-preserving finite volume scheme that can keep both the energy conservation and the second-order accuracy.

The authors in [19] pointed out that any FV scheme based on the harmonic averaging of cell-centered diffusion coefficients will break down when some of these coefficients go to zero or their ratio grows, which results in totally wrong numerical solution profiles in some strongly nonlinear parabolic problems. This phenomenon is referred to as the numerical heat-barrier issue [42]. To solve this problem, a new mimetic scheme with a staggered discretization of diffusion coefficients was then suggested in [19] and analyzed in [26]. The authors in $[23,24]$ presented the discrete schemes with harmonic averaging by adding a correction term for both continuous and discontinuous generalized porous medium equations. In three-temperature RHD, radiation diffusion equations are coupled with hydrodynamics that are usually simulated by Lagrange or ALE algorithms. In this case, the strong nonlinear diffusion coefficients are closely related to some cell-centered quantities such as density. Since the density may be discontinuous across the grid edges in Lagrange hydrodynamics, it's very complicated to define certain diffusion coefficients associated with the edges or vertices. To our best knowledge, there exists no cell-centered or hybrid scheme based on cell-centered diffusion coefficients that can preserve the solution positivity and overcome the numerical heat-barrier issue simultaneously. For cell-centered or hybrid FV schemes, the technique to design a positivity-preserving scheme is quite different from the one that handles the numerical heat-barrier issue.

In this paper, we propose a vertex-centered and positivity-preserving scheme for solving 2-D 3-T equations on arbitrary polygonal meshes. The primary unknowns are defined at the vertices, while the auxiliary unknowns are defined at the cell centers and edge midpoints of the primary mesh. A simple interpolation method is used to 
evaluate the auxiliary unknowns and a procedure is suggested to handle the integrals of the possibly discontinuous parameters. The final nonlinear system is solved by the Anderson acceleration of the Picard method. The kernel of a positivity-preserving FV scheme is the decomposition of the co-normal and the construction of the flux approximation. To our knowledge, most existing positivity-preserving FV schemes use the convex decomposition of the co-normal, which leads to a dynamic stencil for the flux approximation and requires a certain search algorithm (see [20,35-37] and the references therein). Here we adopt a different approach to design a flux approximation with a fixed stencil, avoiding the search algorithm for complex grids. The original idea to build a positivity-preserving FV scheme with a fixed stencil was suggested in $[9,50]$ for single diffusion equation. Moreover, as pointed out in [42], vertex-centered FV schemes with cell-centered diffusion coefficients are generally free of the numerical heat-barrier issue, thus no special treatment is required here.

To summarize, the proposed scheme has the following characteristics:

1. It is a pure vertex-centered scheme and the cell-centered and edge-midpoint unknowns are interpolated by simple algorithms;

2. The co-normal decomposition is fixed, which leads to a fixed stencil of the flux approximation;

3. It is positivity-preserving for the primary unknowns. Accordingly, the auxiliary unknowns in the edge midpoints and the cell centers are positivity-preserving;

4. It works on general polygonal meshes with star-shaped cells for both discontinuous and strong nonlinear radiation diffusion problems;

5. It effectively alleviates the so-called numerical heat-barrier issue in radiation diffusion problems without any special treatment.

The first and fifth features are the main differences from the ones in [9], while the rest ones make it most attractive than those in [7,31,51].

The rest of this paper is organized as follows. In Section 2, a short description of 2-D 3-T radiation diffusion equations is presented. The vertex-centered and positivitypreserving scheme is constructed in Section 3 and in Section 4, the monotonicity of the present scheme is analyzed and the nonlinear iteration method is discussed. Finally, numerical experiments are presented in Section 5 and some concluding remarks are given in the last section.

\section{2-D 3-T radiation diffusion equations}

Consider the 2-D 3-T radiation diffusion equations $[7,14]$ 


$$
\begin{aligned}
& \rho c_{v e} \frac{\partial T_{e}}{\partial t}-\nabla \cdot\left(\kappa_{e} \nabla T_{e}\right)=\rho \omega_{e i}\left(T_{i}-T_{e}\right)+\rho \omega_{e r}\left(T_{r}-T_{e}\right), \\
& \rho c_{v i} \frac{\partial T_{i}}{\partial t}-\nabla \cdot\left(\kappa_{i} \nabla T_{i}\right)=\rho \omega_{e i}\left(T_{e}-T_{i}\right), \\
& c_{v r} \frac{\partial T_{r}^{4}}{\partial t}-\nabla \cdot\left(\kappa_{r} \nabla T_{r}\right)=\rho \omega_{e r}\left(T_{e}-T_{r}\right),
\end{aligned}
$$

where

- $T_{\alpha}, \alpha=e, i, r$ are the temperature functions of electron, ion and photon, respectively;

- $\rho$ denotes the density of the material, which is a constant within each subdomain and discontinuous across interfaces of subdomains;

- $\omega_{e i}=A_{e i} \rho T_{e}^{-2 / 3}$ and $\omega_{e r}=A_{e r} \rho T_{e}^{-1 / 2}$ are the energy exchange coefficients between electron and ion and between electron and photon, respectively;

- $\kappa_{\alpha}=A_{\alpha} T_{\alpha}^{5 / 2}, \alpha=e, i, \kappa_{r}=A_{r} T_{r}^{3+\beta}$ are the diffusion coefficients of electron, ion and photon, respectively;

- $c_{v \alpha}, \alpha=e, i, r$ are the thermal capacities of electron, ion and photon, respectively;

- Parameters $A_{\alpha}, \alpha=e, i, r, \beta, A_{e i}, A_{e r}$ are different constants in different subdomains.

The boundary and initial conditions are

- boundary conditions:

$$
\begin{cases}\kappa_{\alpha} \frac{\partial T_{\alpha}}{\partial \boldsymbol{n}}=0, \quad \alpha=e, i ; \quad T_{r}=g_{D}(\boldsymbol{x}, t), & \text { on free surfaces } \\ \kappa_{\alpha} \frac{\partial T_{\alpha}}{\partial \boldsymbol{n}}=0, \quad \alpha=e, i, r, & \text { on rigid walls }\end{cases}
$$

where $\boldsymbol{n}$ is the unit outer normal vector along boundary and $g_{D}(\boldsymbol{x}, t)$ is a given function.

- initial conditions:

$$
T_{\alpha}=g_{\alpha}(\boldsymbol{x}), \quad \alpha=e, i, r,
$$

where $g_{\alpha}(\boldsymbol{x})$ is a given function.

As a result, (2.1a)-(2.3) becomes a closed and well-posed system [31,33].

This system approximately describes the process of radiant energy spreading in the quiescent medium and energy exchange of electrons with photons and ions. The total energy of unit mass can be defined by

$$
E=E_{e}+E_{i}+E_{r}
$$

where $E_{e}=c_{v e} T_{e}, E_{i}=c_{v i} T_{i}, E_{r}=c_{v r} T_{r}^{4} / \rho$. 


\section{Construction of the positivity-preserving scheme}

\subsection{Mesh notations}

The domain $\Omega$ is decomposed into a number of non-overlapped polygonal cells that form the so-called primary mesh, see the mesh with solid line segments in Fig. 1. The cell center is defined as the geometric center whose coordinates are the simple averages of the cell vertices. Other definitions for cell centers are also allowed, which may result in more complicated interpolation algorithms. Assume that all primary cells are star-shaped with respect to their cell centers, i.e., any ray emanating from the cell center intersects the cell boundary at exactly one point. Then, each primary cell can be further partitioned into several quadrilateral subcells by connecting the cell center with the edge midpoints, see the dashed lined segments in Fig. 1. All subcells sharing the same vertex in the primary mesh form a polygonal cell of the dual mesh. All dual cells are also star-shaped with respect to the corresponding primary vertices.

Here we introduce two categories of unknowns on the primary mesh and its dual counterpart, respectively. The primary unknowns are defined at the vertices of the primary mesh in $\Omega$ or on $\partial \Omega \backslash \Gamma_{D}$ and are shown by solid circles in Fig. 1 where $\Gamma_{D}$ is the Dirichlet boundary. The auxiliary unknowns are defined at the cell centers and edge midpoints of the primary mesh, which are shown by hollow circles and squares in Fig. 1, respectively. Each primary unknown has a FV equation associated with it, while the auxiliary unknowns are evaluated by primary unknowns, or Dirichlet boundary data if necessary, which will be discussed in details later.

To facilitate the exposition, we introduce some notations, some of which are shown in Fig. 2.

- $K$, a generic primary cell, its cell center, measure, diameter, set of edges, number of edges and the constant restriction of $\kappa_{\alpha}$ are denoted as $\boldsymbol{x}_{K},|K|, h_{K}, \mathcal{E}_{K}, n_{K}$ and $\kappa_{\alpha, K}$, respectively.

- $\mathcal{M}$, the set of primary cells in $\bar{\Omega}$ and $h=\max _{K \in \mathcal{M}} h_{K}$ the mesh size.

- $\mathcal{M}_{\nu}$, the set of primary cells sharing the vertex $\boldsymbol{x}_{\nu}$.

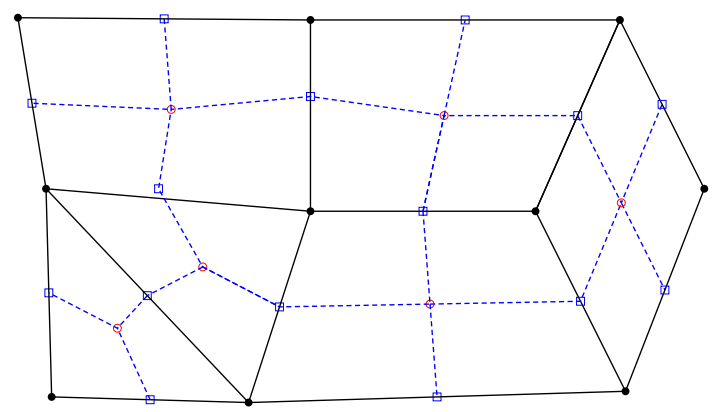

Figure 1: The primary mesh (solid line) and dual mesh (dashed line). 


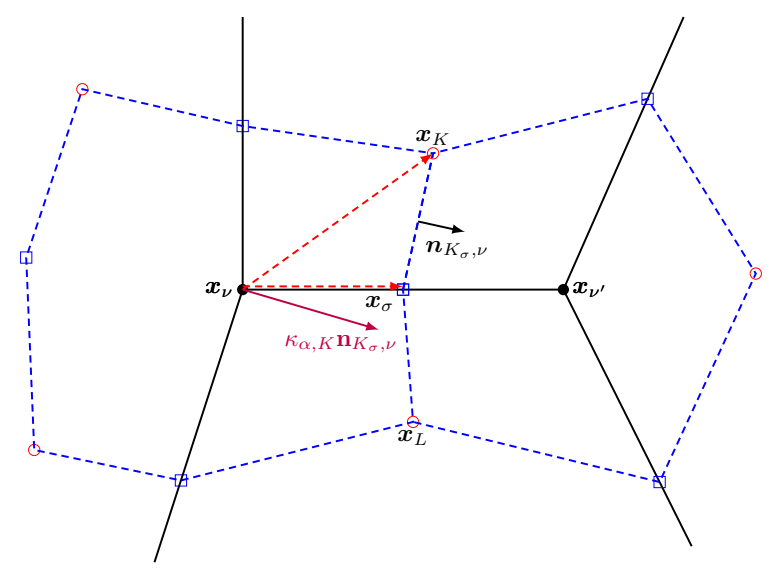

Figure 2: Notations for the construction of one-sided flux.

- $\mathcal{E}_{\nu}$, the set of primary cell edges sharing $\boldsymbol{x}_{\nu}$.

- $\sigma$, a generic primary edge of $K$ with endpoints $\boldsymbol{x}_{\nu}$ and $\boldsymbol{x}_{\nu^{\prime}}$. Its midpoint is denoted as $\boldsymbol{x}_{\sigma}$.

- $K_{\nu}^{*}$, the dual cell corresponding to vertex $\boldsymbol{x}_{\nu}$.

- $\boldsymbol{n}_{K_{\sigma}, \nu}$ (resp. $\boldsymbol{n}_{K_{\sigma}, \nu^{\prime}}$ ), the normal vector to a dual edge $\boldsymbol{x}_{\sigma} \boldsymbol{x}_{K}$ outward to the dual cell associated with $\boldsymbol{x}_{\nu}$ (resp. $\boldsymbol{x}_{\nu^{\prime}}$ ) and $\left\|\boldsymbol{n}_{K_{\sigma}, \nu}\right\|=\left\|\boldsymbol{n}_{K_{\sigma}, \nu^{\prime}}\right\|=\left|\boldsymbol{x}_{\sigma} \boldsymbol{x}_{K}\right|$. Obviously, $\boldsymbol{n}_{K_{\sigma}, \nu}=-\boldsymbol{n}_{K_{\sigma}, \nu^{\prime}}$

\subsection{One-sided flux formulation} obtain

Integrating (2.1a)-(2.1c) over the dual cell $K_{\nu}^{*}$ with respect to $x_{\nu}$, respectively, we

$$
\begin{aligned}
& \int_{K_{\nu}^{*}} \rho c_{v e} \frac{\partial T_{e}}{\partial t} d x d y+\sum_{\boldsymbol{x}_{\sigma} \boldsymbol{x}_{K} \in \partial K_{\nu}^{*}} \mathcal{F}_{K_{\sigma}, \nu}^{e} \\
& =\int_{K_{\nu}^{*}} \rho \omega_{e i}\left(T_{i}-T_{e}\right) d x d y+\int_{K_{\nu}^{*}} \rho \omega_{e r}\left(T_{r}-T_{e}\right) d x d y \\
& \int_{K_{\nu}^{*}} \rho c_{v i} \frac{\partial T_{i}}{\partial t} d x d y+\sum_{\boldsymbol{x}_{\sigma} \boldsymbol{x}_{K} \in \partial K_{\nu}^{*}} \mathcal{F}_{K_{\sigma}, \nu}^{i}=\int_{K_{\nu}^{*}} \rho \omega_{e i}\left(T_{e}-T_{i}\right) d x d y, \\
& \int_{K_{\nu}^{*}} c_{v r} \frac{\partial T_{r}^{4}}{\partial t} d x d y+\sum_{\boldsymbol{x}_{\sigma} \boldsymbol{x}_{K} \in \partial K_{\nu}^{*}} \mathcal{F}_{K_{\sigma}, \nu}^{r}=\int_{K_{\nu}^{*}} \rho \omega_{e r}\left(T_{e}-T_{r}\right) d x d y,
\end{aligned}
$$

where the continuous fluxes on the dual edge $\boldsymbol{x}_{\sigma} \boldsymbol{x}_{K}$ are

$$
\mathcal{F}_{K_{\sigma}, \nu}^{\alpha}=-\int_{\boldsymbol{x}_{\sigma} \boldsymbol{x}_{K}} \kappa_{\alpha} \nabla T_{\alpha} \cdot \boldsymbol{n}_{K_{\sigma}, \nu} d s, \quad \alpha=e, i, r
$$


Next, we will give the discrete approximations of $\mathcal{F}_{K_{\sigma}, \nu}^{\alpha}$.

As shown in Fig. 2, for the normal $\boldsymbol{n}_{K_{\sigma}, \nu}$, we have the following fixed decomposition

$$
\boldsymbol{n}_{K_{\sigma}, \nu}=\beta_{K_{\sigma}, \nu}\left(\boldsymbol{x}_{K}-\boldsymbol{x}_{\nu}\right)+\gamma_{K_{\sigma}, \nu}\left(\boldsymbol{x}_{\sigma}-\boldsymbol{x}_{\nu}\right),
$$

where

$$
\beta_{K_{\sigma}, \nu}=\frac{\boldsymbol{n}_{K_{\sigma}, \nu}^{T} \mathcal{R}\left(\boldsymbol{x}_{\sigma}-\boldsymbol{x}_{\nu}\right)}{\left(\boldsymbol{x}_{K}-\boldsymbol{x}_{\nu}\right)^{T} \mathcal{R}\left(\boldsymbol{x}_{\sigma}-\boldsymbol{x}_{\nu}\right)}, \quad \gamma_{K_{\sigma}, \nu}=\frac{\boldsymbol{n}_{K_{\sigma}, \nu}^{T} \mathcal{R}\left(\boldsymbol{x}_{K}-\boldsymbol{x}_{\nu}\right)}{\left(\boldsymbol{x}_{\sigma}-\boldsymbol{x}_{\nu}\right)^{T} \mathcal{R}\left(\boldsymbol{x}_{K}-\boldsymbol{x}_{\nu}\right)},
$$

and $\mathcal{R}$ denotes an operator that rotates a vector clockwise to its normal direction. Here we remark that the co-normal $\kappa_{\alpha, K} \boldsymbol{n}_{K_{\sigma}, \nu}$ is collinear with the normal $\boldsymbol{n}_{K_{\sigma}, \nu}$ since $\kappa_{\alpha, K}$ is scalar and one of the coefficients in (3.2) may be negative so that it is in general not a convex decomposition. It follows from (3.2) that

$$
\mathcal{F}_{K_{\sigma}, \nu}^{\alpha} \simeq \kappa_{\alpha, K}\left(\beta_{K_{\sigma}, \nu}\left(T_{\alpha}\left(\boldsymbol{x}_{\nu}\right)-T_{\alpha}\left(\boldsymbol{x}_{K}\right)\right)+\gamma_{K_{\sigma}, \nu}\left(T_{\alpha}\left(\boldsymbol{x}_{\nu}\right)-T_{\alpha}\left(\boldsymbol{x}_{\sigma}\right)\right)\right), \quad \alpha=e, i, r,
$$

here and hereafter $\simeq$ indicates that the formula holds in the linearity-preserving sense, i.e., the corresponding formula holds exactly when the solution is piecewise linear and the diffusion coefficient is piecewise constant with respect to the primary mesh. Then we obtain the following one-sided flux approximation with respect to the dual edge $\boldsymbol{x}_{\sigma} \boldsymbol{x}_{K}$

$$
F_{K_{\sigma}, \nu}^{\alpha}=\kappa_{\alpha, K}\left(\beta_{K_{\sigma}, \nu}\left(T_{\alpha, \nu}-T_{\alpha, K}\right)+\gamma_{K_{\sigma}, \nu}\left(T_{\alpha, \nu}-T_{\alpha, \sigma}\right)\right), \quad \alpha=e, i, r,
$$

where $T_{\alpha, K}, T_{\alpha, \nu}$ and $T_{\alpha, \sigma}$ denote the approximations of $T_{\alpha}$ at $\boldsymbol{x}_{K}, \boldsymbol{x}_{\nu}$ and $\boldsymbol{x}_{\sigma}$, respectively.

Analogously, by the vector splitting

$$
\boldsymbol{n}_{K_{\sigma}, \nu^{\prime}}=\beta_{K_{\sigma}, \nu^{\prime}}\left(\boldsymbol{x}_{K}-\boldsymbol{x}_{\nu^{\prime}}\right)+\gamma_{K_{\sigma}, \nu^{\prime}}\left(\boldsymbol{x}_{\sigma}-\boldsymbol{x}_{\nu^{\prime}}\right),
$$

we obtain another one-sided flux approximation

$$
F_{K_{\sigma}, \nu^{\prime}}^{\alpha}=\kappa_{\alpha, K}\left(\beta_{K_{\sigma}, \nu^{\prime}}\left(T_{\alpha, \nu^{\prime}}-T_{\alpha, K}\right)+\gamma_{K_{\sigma}, \nu^{\prime}}\left(T_{\alpha, \nu^{\prime}}-T_{\alpha, \sigma}\right)\right), \quad \alpha=e, i, r,
$$

where $T_{\alpha, \nu^{\prime}}$ denotes the approximation of $T_{\alpha}$ at $\boldsymbol{x}_{\nu^{\prime}}$ and the coefficients in (3.6) are computed by

$$
\beta_{K_{\sigma}, \nu^{\prime}}=\frac{\boldsymbol{n}_{K_{\sigma}, \nu^{\prime}}^{T} \mathcal{R}\left(\boldsymbol{x}_{\sigma}-\boldsymbol{x}_{\nu^{\prime}}\right)}{\left(\boldsymbol{x}_{K}-\boldsymbol{x}_{\nu^{\prime}}\right)^{T} \mathcal{R}\left(\boldsymbol{x}_{\sigma}-\boldsymbol{x}_{\nu^{\prime}}\right)}, \quad \gamma_{K_{\sigma}, \nu^{\prime}}=\frac{\boldsymbol{n}_{K_{\sigma}, \nu^{\prime}}^{T} \mathcal{R}\left(\boldsymbol{x}_{K}-\boldsymbol{x}_{\nu^{\prime}}\right)}{\left(\boldsymbol{x}_{\sigma}-\boldsymbol{x}_{\nu^{\prime}}\right)^{T} \mathcal{R}\left(\boldsymbol{x}_{K}-\boldsymbol{x}_{\nu^{\prime}}\right)} .
$$

Lemma 3.1. Assume that each primary cell is star-shaped with respect to its center. Then for the coefficients in the one-sided fluxes (3.4) and (3.6), we have

$$
\beta_{K_{\sigma}, \nu}+\gamma_{K_{\sigma}, \nu}=\beta_{K_{\sigma}, \nu^{\prime}}+\gamma_{K_{\sigma}, \nu^{\prime}}=\frac{\left|\boldsymbol{x}_{\sigma} \boldsymbol{x}_{K}\right|}{d_{K_{\sigma}, \nu}}
$$

and

$$
\beta_{K_{\sigma}, \nu}+\beta_{K_{\sigma}, \nu^{\prime}}=0,
$$

where $d_{K_{\sigma}, \nu}$ denotes the distance from the vertex $\boldsymbol{x}_{\nu}$ to the dual edge $\boldsymbol{x}_{\sigma} \boldsymbol{x}_{K}$. 
Proof. Rewriting (3.2) and (3.5), we have

$$
\boldsymbol{n}_{K_{\sigma}, \nu}=\beta_{K_{\sigma}, \nu}\left(\boldsymbol{x}_{K}-\boldsymbol{x}_{\sigma}\right)+\left(\beta_{K_{\sigma}, \nu}+\gamma_{K_{\sigma}, \nu}\right)\left(\boldsymbol{x}_{\sigma}-\boldsymbol{x}_{\nu}\right)
$$

and

$$
\boldsymbol{n}_{K_{\sigma}, \nu^{\prime}}=\beta_{K_{\sigma}, \nu^{\prime}}\left(\boldsymbol{x}_{K}-\boldsymbol{x}_{\sigma}\right)+\left(\beta_{K_{\sigma}, \nu^{\prime}}+\gamma_{K_{\sigma}, \nu^{\prime}}\right)\left(\boldsymbol{x}_{\sigma}-\boldsymbol{x}_{\nu^{\prime}}\right) .
$$

Note that $\boldsymbol{n}_{K_{\sigma}, \nu}=-\boldsymbol{n}_{K_{\sigma}, \nu^{\prime}}=\mathcal{R}\left(\boldsymbol{x}_{K}-\boldsymbol{x}_{\sigma}\right)$ and $\boldsymbol{x}_{\sigma}-\boldsymbol{x}_{\nu^{\prime}}=-\left(\boldsymbol{x}_{\sigma}-\boldsymbol{x}_{\nu}\right)$. We deduce that

$$
\mathbf{0}=\left(\beta_{K_{\sigma}, \nu}+\beta_{K_{\sigma}, \nu^{\prime}}\right)\left(\boldsymbol{x}_{K}-\boldsymbol{x}_{\sigma}\right)+\left(\beta_{K_{\sigma}, \nu}+\gamma_{K_{\sigma}, \nu}-\beta_{K_{\sigma}, \nu^{\prime}}-\gamma_{K_{\sigma}, \nu^{\prime}}\right)\left(\boldsymbol{x}_{\sigma}-\boldsymbol{x}_{\nu}\right)
$$

and

$$
\begin{gathered}
\beta_{K_{\sigma}, \nu}+\gamma_{K_{\sigma}, \nu}=\frac{\boldsymbol{n}_{K_{\sigma}, \nu}^{T} \mathcal{R}\left(\boldsymbol{x}_{K}-\boldsymbol{x}_{\sigma}\right)}{\left(\boldsymbol{x}_{\sigma}-\boldsymbol{x}_{\nu}\right)^{T} \mathcal{R}\left(\boldsymbol{x}_{K}-\boldsymbol{x}_{\sigma}\right)} \\
=\frac{\left|\boldsymbol{x}_{\sigma} \boldsymbol{x}_{K}\right|^{2}}{\left(P \boldsymbol{x}_{\nu}-\boldsymbol{x}_{\nu}\right)^{T} \mathcal{R}\left(\boldsymbol{x}_{K}-\boldsymbol{x}_{\sigma}\right)}=\frac{\left|\boldsymbol{x}_{\sigma} \boldsymbol{x}_{K}\right|}{d_{K_{\sigma}, \nu}},
\end{gathered}
$$

where $P \boldsymbol{x}_{\nu}$ denotes the orthogonal projection point of $\boldsymbol{x}_{\nu}$ onto the line connecting $\boldsymbol{x}_{\sigma}$ and $\boldsymbol{x}_{K}$. Since $\boldsymbol{x}_{K}-\boldsymbol{x}_{\sigma}$ and $\boldsymbol{x}_{\sigma}-\boldsymbol{x}_{\nu}$ are not collinear, we reach (3.7) and (3.8), which completes the proof.

\subsection{The unique flux approximation}

For the interior dual edge $\boldsymbol{x}_{\sigma} \boldsymbol{x}_{K}$, we define a new and unique flux as a linear combination of the one-sided fluxes (3.4) and (3.6), i.e.,

$$
\widetilde{F}_{K_{\sigma}, \nu}^{\alpha}=\mu_{\alpha, 1} F_{K_{\sigma}, \nu}^{\alpha}-\mu_{\alpha, 2} F_{K_{\sigma}, \nu^{\prime}}^{\alpha}, \quad \widetilde{F}_{K_{\sigma}, \nu^{\prime}}^{\alpha}=\mu_{\alpha, 2} F_{K_{\sigma}, \nu^{\prime}}^{\alpha}-\mu_{\alpha, 1} F_{K_{\sigma}, \nu}^{\alpha},
$$

where $\mu_{\alpha, 1}$ and $\mu_{\alpha, 2}$ are two nonnegative parameters, satisfying

$$
\mu_{\alpha, 1}+\mu_{\alpha, 2}=1
$$

Obviously, (3.9) satisfies the local conservation condition. Substituting (3.4) and (3.6) into the first equation of (3.9) and rearranging the terms, we have

$$
\widetilde{F}_{K_{\sigma}, \nu}^{\alpha}=\kappa_{\alpha, K}\left(\mu_{\alpha, 1}\left(\beta_{K_{\sigma}, \nu}+\gamma_{K_{\sigma}, \nu}\right) T_{\alpha, \nu}-\mu_{\alpha, 2}\left(\beta_{K_{\sigma}, \nu^{\prime}}+\gamma_{K_{\sigma}, \nu^{\prime}}\right) T_{\alpha, \nu^{\prime}}+B_{\alpha, K_{\sigma}}\right),
$$

where

$$
B_{\alpha, K_{\sigma}}=\mu_{\alpha, 2} \xi_{\alpha, 2}-\mu_{\alpha, 1} \xi_{\alpha, 1}
$$

and

$$
\xi_{\alpha, 1}=\beta_{K_{\sigma}, \nu} T_{\alpha, K}+\gamma_{K_{\sigma}, \nu} T_{\alpha, \sigma}, \quad \xi_{\alpha, 2}=\beta_{K_{\sigma}, \nu^{\prime}} T_{\alpha, K}+\gamma_{K_{\sigma}, \nu^{\prime}} T_{\alpha, \sigma}
$$

We choose

$$
\mu_{\alpha, 1}= \begin{cases}0.5, & \xi_{\alpha, 1}=\xi_{\alpha, 2}=0 \\ \frac{\left|\xi_{\alpha, 2}\right|}{\left|\xi_{\alpha, 1}\right|+\left|\xi_{\alpha, 2}\right|}, & \text { otherwise }\end{cases}
$$


and $\mu_{\alpha, 2}=1-\mu_{\alpha, 1}$. A direct consequence of the choice of (3.13) is $B_{\alpha, K_{\sigma}}=0$ if $\xi_{\alpha, 1} \xi_{\alpha, 2} \geq 0$. Denote

$$
B_{\alpha, K_{\sigma}}^{+}=\frac{\left|B_{\alpha, K_{\sigma}}\right|+B_{\alpha, K_{\sigma}}}{2}, \quad B_{\alpha, K_{\sigma}}^{-}=\frac{\left|B_{\alpha, K_{\sigma}}\right|-B_{\alpha, K_{\sigma}}}{2},
$$

and $\epsilon$ a positive number which is up to machine precision. Then, $B_{\alpha, K_{\sigma}}$ can be rewritten as

$$
B_{\alpha, K_{\sigma}}=\frac{T_{\alpha, \nu}}{T_{\alpha, \nu}+\epsilon} B_{\alpha, K_{\sigma}}^{+}-\frac{T_{\alpha, \nu^{\prime}}}{T_{\alpha, \nu^{\prime}}+\epsilon} B_{\alpha, K_{\sigma}}^{-}+B_{\alpha, K_{\sigma}}^{\epsilon},
$$

where

$$
B_{\alpha, K_{\sigma}}^{\epsilon}=\frac{\epsilon}{T_{\alpha, \nu}+\epsilon} B_{\alpha, K_{\sigma}}^{+}-\frac{\epsilon}{T_{\alpha, \nu^{\prime}}+\epsilon} B_{\alpha, K_{\sigma}}^{-} .
$$

It follows from (3.11) that

$$
\widetilde{F}_{K_{\sigma}, \nu}^{\alpha}=A_{\alpha, K_{\sigma}} T_{\alpha, \nu}-A_{\alpha, K_{\sigma^{\prime}}} T_{\alpha, \nu^{\prime}}+\kappa_{\alpha, K} B_{\alpha, K_{\sigma}}^{\epsilon},
$$

where

$$
\begin{aligned}
& A_{\alpha, K_{\sigma}}=\kappa_{\alpha, K}\left(\mu_{\alpha, 1}\left(\beta_{K_{\sigma}, \nu}+\gamma_{K_{\sigma}, \nu}\right)+\frac{B_{\alpha, K_{\sigma}}^{+}}{T_{\alpha, \nu}+\epsilon}\right), \\
& A_{\alpha, K_{\sigma^{\prime}}}=\kappa_{\alpha, K}\left(\mu_{\alpha, 2}\left(\beta_{K_{\sigma}, \nu^{\prime}}+\gamma_{K_{\sigma}, \nu^{\prime}}\right)+\frac{B_{\alpha, K_{\sigma}}^{-}}{T_{\alpha, \nu^{\prime}}+\epsilon}\right) .
\end{aligned}
$$

$B_{\alpha, K_{\sigma}}^{\epsilon}$ is a second-order term under some assumptions, which can be proved in a way similar to that of (29) in [50] and it is omitted here. Thus, by truncating $\kappa_{\alpha, K} B_{\alpha, K_{\sigma}}^{\epsilon}$ in (3.14) and by (3.9), we reach a new definition of the unique edge flux, given by

$$
\widetilde{F}_{K_{\sigma}, \nu}^{\alpha}=A_{\alpha, K_{\sigma}} T_{\alpha, \nu}-A_{\alpha, K_{\sigma^{\prime}}} T_{\alpha, \nu^{\prime}}, \quad \widetilde{F}_{K_{\sigma}, \nu^{\prime}}^{\alpha}=A_{\alpha, K_{\sigma^{\prime}}} T_{\alpha, \nu^{\prime}}-A_{\alpha, K_{\sigma}} T_{\alpha, \nu} .
$$

Obviously, the local conservation condition is still maintained. Moreover, if $T_{\alpha} \geq 0$, $A_{\alpha, K_{\sigma}}$ and $A_{\alpha, K_{\sigma^{\prime}}}$ are apparently nonnegative by (3.7), which is the basis of the entire formulation to maintain the positivity-preserving property.

In the case of the dual edge $\sigma$ located in the Neumann boundary, it deduced from (2.2) that

$$
\widetilde{F}_{K_{\sigma}, \nu}^{\alpha}=-\int_{\sigma} \kappa_{\alpha} \frac{\partial T_{\alpha}}{\partial \boldsymbol{n}} d l=0
$$

\subsection{The expression of auxiliary unknowns}

In the present scheme, the auxiliary unknowns are defined at centers and edge midpoints of the primary cells, which can be easily interpolated by the vertex unknowns. Let $\boldsymbol{x}_{\sigma}$ be the midpoint of $\sigma$ whose endpoints are $\boldsymbol{x}_{\nu}$ and $\boldsymbol{x}_{\nu^{\prime}}, \boldsymbol{x}_{K}$ is the geometric center of $K$. Then, for the auxiliary unknowns defined at $\boldsymbol{x}_{\sigma}$ and $\boldsymbol{x}_{K}$, we have

$$
T_{\alpha, \sigma}=\frac{1}{2}\left(T_{\alpha, \nu}+T_{\alpha, \nu^{\prime}}\right), \quad T_{\alpha, K}=\frac{1}{n_{K}} \sum_{\boldsymbol{x}_{\nu} \in \mathcal{V}_{K}} T_{\alpha, \nu}, \quad \alpha=e, i, r,
$$


where $\mathcal{V}_{K}$ denotes the set of vertices in $K$. If $\boldsymbol{x}_{K}$ is not the geometric center of $K$, we adopt the second-order and positivity-preserving interpolation algorithm for the cell-centered unknowns suggested in Subsection 3.5 of [42].

\subsection{The final nonlinear scheme}

Since the parameters $\rho, c_{v \alpha}(\alpha=e, i, r), \omega_{e \alpha}(\alpha=i, r)$ are possibly discontinuous across the interfaces of different material areas, the approximate values of these parameters inside the dual cells need to be considered specially. Here we suggest the strategy in [7] to deal with these parameters. Let $\eta$ be a possibly discontinuous parameter, then the restriction of $\eta$ on the dual cell $K_{\nu}^{*}$ is given by

$$
\bar{\eta}_{K_{\nu}^{*}}:=\frac{1}{\left|K_{\nu}^{*}\right|} \int_{K_{\nu}^{*}} \eta(x, y) d x d y \approx \frac{1}{\left|K_{\nu}^{*}\right|} \sum_{K \in \mathcal{M}_{\nu}} \eta\left(\boldsymbol{x}_{K}\right)\left|K_{\nu}^{*} \cap K\right|
$$

If $\eta$ is nonlinear with respect to $T_{\alpha}$, (3.19) is updated by

$$
\bar{\eta}_{K_{\nu}^{*}} \approx \frac{1}{\left|K_{\nu}^{*}\right|} \sum_{K \in \mathcal{M}_{\nu}} \eta\left(\boldsymbol{x}_{K}, T_{\alpha, K}\right)\left|K_{\nu}^{*} \cap K\right|
$$

where $T_{\alpha, K}$ is the value of $T_{\alpha}$ at the cell center of $K$. In the following, the parameter $\bar{\eta}_{K_{\nu}^{*}}$ is abbreviated as $\bar{\eta}$ whenever there is no confusion.

For the temporal discretization, we use the backward Euler method to ensure the property of numerical stability. With the definition of $\widetilde{F}_{K_{\sigma}, \nu}^{\alpha}$ in (3.16) and (3.17), the finite volume equations with respect to a vertex $\boldsymbol{x}_{\nu} \in \bar{\Omega} \backslash \Gamma_{D}$ are constructed as follows:

$$
\begin{aligned}
& \overline{\rho c_{v e}}\left|K_{\nu}^{*}\right| \frac{T_{e, \nu}^{n+1}-T_{e, \nu}^{n}}{\tau}+\sum_{K \in \mathcal{M}_{\nu}} \sum_{\sigma \subset \mathcal{E}_{K} \cap \mathcal{E}_{\nu}} \widetilde{F}_{K_{\sigma}, \nu}^{e, n+1} \\
& =\bar{\rho}\left|K_{\nu}^{*}\right|\left({\overline{\omega_{e i}}}^{n+1}\left(T_{i, \nu}^{n+1}-T_{e, \nu}^{n+1}\right)+{\overline{\omega_{e r}}}^{n+1}\left(T_{r, \nu}^{n+1}-T_{e, \nu}^{n+1}\right)\right), \\
& \overline{\rho c_{v i}}\left|K_{\nu}^{*}\right| \frac{T_{i, \nu}^{n+1}-T_{i, \nu}^{n}}{\tau}+\sum_{K \in \mathcal{M}_{\nu}} \sum_{\sigma \subset \mathcal{E}_{K} \cap \mathcal{E}_{\nu}} \widetilde{F}_{K_{\sigma}, \nu}^{i, n+1}=\bar{\rho}\left|K_{\nu}^{*}\right| \bar{\omega}_{e i}^{n+1}\left(T_{e, \nu}^{n+1}-T_{i, \nu}^{n+1}\right), \\
& 4 \overline{c_{v r}}\left|K_{\nu}^{*}\right|\left(T_{i, \nu}^{n+1}\right)^{3} \frac{T_{i, \nu}^{n+1}-T_{i, \nu}^{n}}{\tau}+\sum_{K \in \mathcal{M}_{\nu}} \sum_{\sigma \subset \mathcal{E}_{K} \cap \mathcal{E}_{\nu}} \widetilde{F}_{K_{\sigma}, \nu}^{r, n+1} \\
& =\bar{\rho}\left|K_{\nu}^{*}\right| \bar{\omega}_{e r}^{n+1}\left(T_{e, \nu}^{n+1}-T_{r, \nu}^{n+1}\right),
\end{aligned}
$$

where $\tau$ is the time step. Here $\bar{\rho}, \overline{c_{v \alpha}}$ and ${\overline{\omega_{e \alpha}}}^{n+1}(\alpha=i, r)$ are computed by (3.19) or (3.20). 
A Vertex-Centered Positivity-Preserving Scheme for 2-D 3-T Equations

\section{Monotonicity and nonlinear iteration method}

Substituting the expression of flux (3.16) and (3.17) into (3.21a)-(3.21c), we have

$$
\begin{aligned}
& \overline{\rho c_{v e}}\left|K_{\nu}^{*}\right| \frac{T_{e, \nu}^{n+1}-T_{e, \nu}^{n}}{\tau}+\sum_{K \in \mathcal{M}_{\nu}} \sum_{\sigma \subset \mathcal{E}_{K} \cap \mathcal{E}_{\nu}}\left(A_{e, K_{\sigma}}^{n+1} T_{e, \nu}^{n+1}-A_{e, K_{\sigma^{\prime}}}^{n+1} T_{e, \nu^{\prime}}^{n+1}\right) \\
& =\bar{\rho}\left|K_{\nu}^{*}\right|\left({\overline{\omega_{e i}}}^{n+1}\left(T_{i, \nu}^{n+1}-T_{e, \nu}^{n+1}\right)+\bar{\omega}_{e r}^{n+1}\left(T_{r, \nu}^{n+1}-T_{e, \nu}^{n+1}\right)\right), \\
& \overline{\rho c_{v i}}\left|K_{\nu}^{*}\right| \frac{T_{i, \nu}^{n+1}-T_{i, \nu}^{n}}{\tau}+\sum_{K \in \mathcal{M}_{\nu}} \sum_{\sigma \subset \mathcal{E}_{K} \cap \mathcal{E}_{\nu}}\left(A_{i, K_{\sigma}}^{n+1} T_{i, \nu}^{n+1}-A_{i, K_{\sigma^{\prime}}}^{n+1} T_{i, \nu^{\prime}}^{n+1}\right) \\
& =\bar{\rho}\left|K_{\nu}^{*}\right|{\overline{\omega_{e i}}}^{n+1}\left(T_{e, \nu}^{n+1}-T_{i, \nu}^{n+1}\right), \\
& 4 \overline{c_{v r}}\left|K_{\nu}^{*}\right|\left(T_{i, \nu}^{n+1}\right)^{3} \frac{T_{i, \nu}^{n+1}-T_{i, \nu}^{n}}{\tau}+\sum_{K \in \mathcal{M}_{\nu}} \sum_{\sigma \subset \mathcal{E}_{K} \cap \mathcal{E}_{\nu}}\left(A_{r, K_{\sigma}}^{n+1} T_{r, \nu}^{n+1}-A_{r, K_{\sigma^{\prime}}}^{n+1} T_{r, \nu^{\prime}}^{n+1}\right) \\
& =\bar{\rho}\left|K_{\nu}^{*}\right| \bar{\omega}_{e r} n+1\left(T_{e, \nu}^{n+1}-T_{r, \nu}^{n+1}\right) .
\end{aligned}
$$

We use the Picard iteration method to solve the nonlinear system of equations, which is required in maintaining the M-matrix structure of the resulting coefficient matrix. Specifically, we set

$$
\begin{aligned}
& \left(T_{i, \nu}^{n+1}\right)^{3}=\left(T_{i, \nu}^{n+1, k}\right)^{3}, \\
& A_{\alpha, K_{\sigma}}^{n+1}=A_{\alpha, K_{\sigma}}^{n+1, k}, \quad A_{\alpha, K_{\sigma^{\prime}}}^{n+1}=A_{\alpha, K_{\sigma^{\prime}}}^{n+1, k}, \\
& {\overline{\omega_{e r}}}^{n+1}={\overline{\omega_{e r}}}^{n+1, k}, \quad{\overline{\omega_{e i}}}^{n+1}={\overline{\omega_{e i}}}^{n+1, k},
\end{aligned}
$$

where $k$ is the nonlinear iteration index. As a result, we have

$$
\begin{aligned}
& \overline{\rho c_{v e}}\left|K_{\nu}^{*}\right| \frac{T_{e, \nu}^{n+1, k+1}-T_{e, \nu}^{n}}{\tau}+\sum_{K \in \mathcal{M}_{\nu}} \sum_{\sigma \subset \mathcal{E}_{K} \cap \mathcal{E}_{\nu}}\left(A_{e, K_{\sigma}}^{n+1, k} T_{e, \nu}^{n+1, k+1}-A_{e, K_{\sigma^{\prime}}}^{n+1, k} T_{e, \nu^{\prime}}^{n+1, k+1}\right) \\
& =\bar{\rho}\left|K_{\nu}^{*}\right|\left({\overline{\omega_{e i}}}^{n+1, k}\left(T_{i, \nu}^{n+1, k+1}-T_{e, \nu}^{n+1, k+1}\right)+\bar{\omega}_{e r}^{n+1, k}\left(T_{r, \nu}^{n+1, k+1}-T_{e, \nu}^{n+1, k+1}\right)\right), \\
& \overline{\rho c_{v i}}\left|K_{\nu}^{*}\right| \frac{T_{i, \nu}^{n+1, k+1}-T_{i, \nu}^{n}}{\tau}+\sum_{K \in \mathcal{M}_{\nu}} \sum_{\sigma \subset \mathcal{E}_{K} \cap \mathcal{E}_{\nu}}\left(A_{i, K_{\sigma}}^{n+1, k} T_{i, \nu}^{n+1, k+1}-A_{i, K_{\sigma^{\prime}}}^{n+1, k} T_{i, \nu^{\prime}}^{n+1, k+1}\right) \\
& =\bar{\rho}\left|K_{\nu}^{*}\right|{\overline{\omega_{e i}}}^{n+1, k}\left(T_{e, \nu}^{n+1, k+1}-T_{i, \nu}^{n+1, k+1}\right), \\
& 4 \overline{c_{v r}}\left|K_{\nu}^{*}\right|\left(T_{r, \nu}^{n+1, k}\right)^{3} \frac{T_{r, \nu}^{n+1, k+1}-T_{r, \nu}^{n}}{\tau}+\sum_{K \in \mathcal{M}_{\nu}} \sum_{\sigma \subset \mathcal{E}_{K} \cap \mathcal{E}_{\nu}}\left(A_{r, K_{\sigma}}^{n+1, k} T_{r, \nu}^{n+1, k+1}-A_{r, K_{\sigma^{\prime}}}^{n+1, k} T_{r, \nu^{\prime}}^{n+1, k+1}\right) \\
& =\bar{\rho}\left|K_{\nu}^{*}\right| \bar{\omega}_{e r}^{n+1, k}\left(T_{e, \nu}^{n+1, k+1}-T_{r, \nu}^{n+1, k+1}\right) .
\end{aligned}
$$


The above equations can be rewritten as

$$
\begin{aligned}
\bar{\rho}\left(\overline{c_{v e}}\right. & \left.+\tau{\overline{\omega_{e i}}}^{n+1, k}+\tau{\overline{\omega_{e r}}}^{n+1, k}\right)\left|K_{\nu}^{*}\right| T_{e, \nu}^{n+1, k+1} \\
& -\tau \overline{\rho \omega_{e i}}{ }^{n+1, k}\left|K_{\nu}^{*}\right| T_{i, \nu}^{n+1, k+1}-\tau \overline{\rho \omega_{e r}}{ }^{n+1, k}\left|K_{\nu}^{*}\right| T_{r, \nu}^{n+1, k+1} \\
& +\tau \sum_{K \in \mathcal{M}_{\nu}} \sum_{\sigma \subset \mathcal{E}_{K} \cap \mathcal{E}_{\nu}}\left(A_{e, K_{\sigma}}^{n+1, k} T_{e, \nu}^{n+1, k+1}-A_{e, K_{\sigma^{\prime}}}^{n+1, k} T_{e, \nu^{\prime}}^{n+1, k+1}\right)=\overline{\rho c_{v e}}\left|K_{\nu}^{*}\right| T_{e, \nu}^{n}, \\
\bar{\rho}\left(\overline{c_{v i}}\right. & \left.+\tau{\overline{\omega_{e i}}}^{n+1, k}\right)\left|K_{\nu}^{*}\right| T_{i, \nu}^{n+1, k+1}-\tau{\overline{\rho \omega_{e i}}}^{n+1, k}\left|K_{\nu}^{*}\right| T_{e, \nu}^{n+1, k+1} \\
& +\tau \sum_{K \in \mathcal{M}_{\nu}} \sum_{\sigma \subset \mathcal{E}_{K} \cap \mathcal{E}_{\nu}}\left(A_{i, K_{\sigma}}^{n+1, k} T_{i, \nu}^{n+1, k+1}-A_{i, K_{\sigma^{\prime}}}^{n+1, k} T_{i, \nu^{\prime}}^{n+1, k+1}\right)=\overline{\rho c_{v i}}\left|K_{\nu}^{*}\right| T_{i, \nu}^{n}, \\
\left(4 \overline{c_{v r}}\left(T_{r, \nu}^{n+1, k}\right)^{3}+\tau{\overline{\rho \omega_{e r}}}^{n+1, k}\right)\left|K_{\nu}^{*}\right| T_{r, \nu}^{n+1, k+1}-\tau{\overline{\rho \omega_{e r}}}^{n+1, k}\left|K_{\nu}^{*}\right| T_{e, \nu}^{n+1, k+1} & \\
& +\tau \sum_{K \in \mathcal{M}_{\nu}} \sum_{\sigma \subset \mathcal{E}_{K} \cap \mathcal{E}_{\nu}}\left(A_{r, K_{\sigma}}^{n+1, k} T_{r, \nu}^{n+1, k+1}-A_{r, K_{\sigma^{\prime}}}^{n+1, k} T_{r, \nu^{\prime}}^{n+1, k+1}\right) \\
= & 4 \overline{c_{v r}}\left|K_{\nu}^{*}\right| T_{r, \nu}^{n}\left(T_{r, \nu}^{n+1, k}\right)^{3} .
\end{aligned}
$$

Denote by $N$ the number of vertices that belong to $\bar{\Omega} \backslash \Gamma_{D}$. Let

$$
\begin{aligned}
& \boldsymbol{U}^{n}=\left(T_{e, 1}^{n}, T_{i, 1}^{n}, T_{r, 1}^{n}, T_{e, 2}^{n}, \cdots, T_{r, N}^{n}\right)^{T}, \\
& \boldsymbol{U}^{n+1, k}=\left(T_{e, 1}^{n+1, k}, T_{i, 1}^{n+1, k}, T_{r, 1}^{n+1, k}, T_{e, 2}^{n+1, k}, \cdots, T_{r, N}^{n+1, k}\right)^{T},
\end{aligned}
$$

be the discrete unknown vectors with length $3 N$. If $\boldsymbol{U}^{n}$ and $\boldsymbol{U}^{n+1, k}$ are known, (4.1a)(4.1c) form a linear algebraic system with coefficient matrix $\mathbb{P}\left(\boldsymbol{U}^{n+1, k}\right)$, given by

$$
\mathbb{P}\left(\boldsymbol{U}^{n+1, k}\right) \boldsymbol{U}^{n+1, k+1}=\boldsymbol{G}\left(\boldsymbol{U}^{n}, \boldsymbol{U}^{n+1, k}\right) .
$$

Obviously, $\boldsymbol{G}\left(\boldsymbol{U}^{n}, \boldsymbol{U}^{n+1, k}\right)$ is a nonnegative vector. The matrix $\mathbb{P}\left(\boldsymbol{U}^{n+1, k}\right)$ is nonsymmetric and has the following properties:

1. All diagonal entries of matrix $\mathbb{P}\left(\boldsymbol{U}^{n+1, k}\right)$ are positive.

2. All off-diagonal entries of $\mathbb{P}\left(\boldsymbol{U}^{n+1, k}\right)$ are non-positive.

3. Each column sum in $\mathbb{P}\left(\boldsymbol{U}^{n+1, k}\right)$ is positive.

4. The matrix $\mathbb{P}\left(\boldsymbol{U}^{n+1, k}\right)$ is irreducible.

Hence, the transpose of $\mathbb{P}\left(\boldsymbol{U}^{n+1, k}\right)$ is an M-matrix so that $\mathbb{P}^{-1}\left(\boldsymbol{U}^{n+1, k}\right)>0$ (see Corollary 3.20 in [40]), which implies $\boldsymbol{U}^{n+1, k+1} \geq 0$ for all $n \geq 0$ and $k \geq 0$. Therefore, we have proved the following theorem of monotonicity.

Theorem 4.1. Let $\boldsymbol{U}^{0} \geq 0$ and linear systems in Picard iterations be solved exactly. Then all $\boldsymbol{U}^{n+1, k}$ are nonnegative vectors, i.e.,

$$
\boldsymbol{U}^{n+1, k} \geq 0, \quad \forall n \geq 0, \quad k \geq 0 .
$$


For the large-scale calculation of the nonlinear systems, the efficiency of nonlinear solver is another important issue. In [13], an extremum-preserving iterative procedure based on domain decomposition method was suggested for the imperfect interface problem. The Newton-Krylov method was employed to solve the discrete equations for 2-D 3-T radiation equations in $[2,10]$. However, this method does not keep the Mmatrix structure so that the positivity-preserving property may be destroyed. At the present, only the Picard method or fixed-point iteration method can be used because it preserves the M-matrix structure of the coefficient matrix. In some extreme cases such as largely distorted meshes, Picard method converges very slow, which motivates us to seek an efficient nonlinear solver that does not spoil the M-matrix structure of the coefficient matrix. Anderson acceleration is a method to accelerate the fixed-point iteration which stores several prior evaluations of the fixed-point map and computes the new iteration as a linear combination of those evaluations. The method was first proposed by Anderson in [3] and has been applied successfully in many problems. In [1], Anderson acceleration was employed to solve the finite difference scheme of threetemperature energy equations and two strategies were used to improve the robustness of the Anderson-accelerated Picard method. To improve the computational efficiency, Anderson acceleration of the Picard method are used in the following tests.

\section{Numerical experiments}

Define the discrete $L^{2}$-norm and $H^{1}$-norm to evaluate discretization errors, i.e.,

$$
\left\|\varepsilon_{\mathcal{M}}\right\|_{0}=\left(\sum_{\boldsymbol{x}_{\nu} \in \bar{\Omega}}\left|K_{\nu}^{*}\right|\left(\sum_{\alpha=e, i, r}\left(T_{\alpha}\left(\boldsymbol{x}_{\nu}\right)-T_{\alpha, \nu}\right)\right)^{2}\right)^{1 / 2}
$$

and

$$
\left\|\varepsilon_{\mathcal{M}}\right\|_{1}=\left(\sum_{K \in \mathcal{M}} \sum_{\sigma \in \mathcal{E}_{K}} \sum_{\alpha=e, i, r}\left(\left(T_{\alpha}\left(\boldsymbol{x}_{\nu}\right)-T_{\alpha}\left(\boldsymbol{x}_{\nu^{\prime}}\right)\right)-\left(T_{\alpha, \nu}-T_{\alpha, \nu^{\prime}}\right)\right)^{2}\right)^{1 / 2} .
$$

The rate of convergence $R_{\alpha}$ ( $\alpha=u$ for $L^{2}$-norm, $q$ for $H^{1}$-norm) is obtained by a least squares fit on the ones computed on each two successive meshes by the following formula

$$
R_{\alpha}=\frac{\log \left[E_{\alpha}\left(h_{2}\right) / E_{\alpha}\left(h_{1}\right)\right]}{\log \left(h_{2} / h_{1}\right)},
$$

where $h_{1}, h_{2}$ denote the mesh sizes of the two successive meshes and $E_{\alpha}\left(h_{1}\right), E_{\alpha}\left(h_{2}\right)$ the corresponding discrete errors. All tests are performed in double precision, Biconjugate gradient stabilized method (Bi-CGSTAB) suggested in [39] is used for solving linear systems with stopping tolerance $10^{-15}$, while the nonlinear iteration is carried out by the Picard method or its Anderson acceleration with stopping tolerance $10^{-6}$. In addition, we choose $\epsilon=10^{-10}$ in (3.15).

Throughout this section, the notations below will be used in the numerical tests. 
- nunkw: number of primary unknowns;

- ms: maximal stencil that equals to the maximal number of non-zero row entries of the linear system of equations;

- as: averaging stencil that equals to the ratio between the total number of nonzero entries in the linear system of equations and nunkw;

- umin: minimal value of the approximate solution at mesh level $i$;

- umax: maximal value of the approximate solution at mesh level $i$;

- $\overline{\text { iter: }}$ : the average value of number of iterations by the Picard method at mesh level $i$;

- $\overline{\text { iter }}^{A}$ : the average value of number of iterations by Anderson acceleration at mesh level $i$.

\subsection{Convergence verification}

In this part, we verify the convergence of the present scheme by two radiation diffusion problems having non-zero source terms and the analytic solutions.

\subsubsection{Smooth diffusion coefficients}

Here we consider a smooth radiation diffusion problem on $\Omega=[0,1]^{2}$ with full Dirichlet boundary condition. Let parameters in the three temperatures diffusion model of (2.1a)-(2.1c) be specified by

$$
\begin{cases}\rho=1 & \\ \omega_{e i}=T_{e}^{3}, \quad \omega_{e r}=T_{e}, & \\ c_{v \alpha}=2 T_{\alpha}^{2}+26 T_{\alpha}+2, & \alpha=i, e, r \\ \kappa_{\alpha}=1+T_{\alpha}^{2}, & \alpha=i, e, r\end{cases}
$$

The associated analytical solutions are

$$
T_{\alpha}=t+x^{2}+3 y+1, \quad \alpha=e, i, r
$$

Here we remark that the source terms can be computed correspondingly. We choose the final time $t=0.1$ and the time interval $\tau=0.4 \times h^{2}$. Four mesh types are used in this test, see Fig. 3.

Numerical results are presented in Tables 1-4. One can see that the $L^{2}$ error is approximately second order while the $H^{1}$ error achieves about first order. In addition, the present scheme has a local stencil, an eleven-point one on structured quadrilateral meshes. 


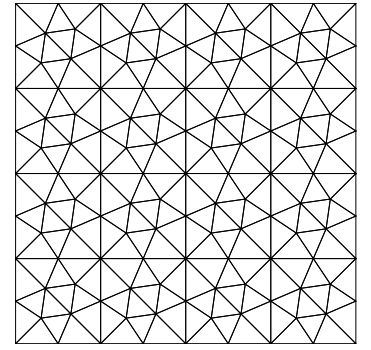

(a) Mesh1: Triangular mesh

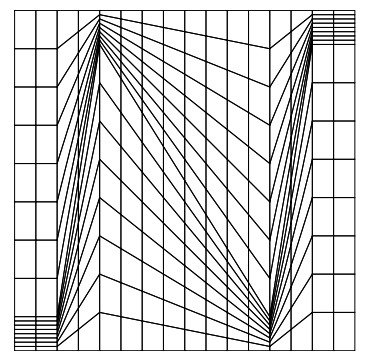

(c) Mesh3: Kershaw mesh

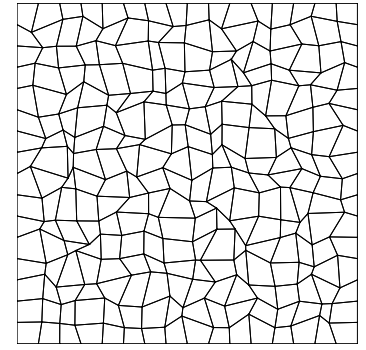

(b) Mesh2: Random mesh

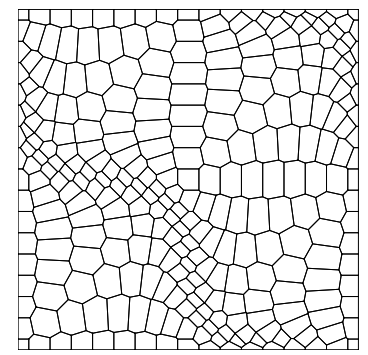

(d) Mesh4: Polygonal mesh

Figure 3: Four mesh types used in the numerical tests.

Table 1: Numerical results of $T_{e}$ on triangular meshes at $t=0.1$.

\begin{tabular}{||ccccccccc||}
\hline Mesh level & numkw & $\mathrm{ms}$ & as & $E_{u}$ & $R_{u}$ & $E_{q}$ & $R_{q}$ & $\overline{\text { iter }}$ \\
\hline 1 & 63 & 11 & 7.19 & $1.07 \mathrm{e}-3$ & - & $1.97 \mathrm{e}-2$ & - & 5.00 \\
2 & 291 & 11 & 8.11 & $5.20 \mathrm{e}-4$ & 1.04 & $1.85 \mathrm{e}-2$ & 0.09 & 5.00 \\
3 & 1251 & 11 & 8.56 & $1.48 \mathrm{e}-4$ & 1.81 & $1.05 \mathrm{e}-2$ & 0.82 & 4.95 \\
4 & 5187 & 11 & 8.78 & $3.82 \mathrm{e}-5$ & 1.95 & $5.40 \mathrm{e}-3$ & 0.96 & 5.00 \\
5 & 21123 & 11 & 8.89 & $9.68 \mathrm{e}-6$ & 1.98 & $2.73 \mathrm{e}-3$ & 0.98 & 5.00 \\
\hline
\end{tabular}

\subsubsection{Discontinuous diffusion tensors}

A radiation diffusion system with discontinuous diffusion tensors on $\Omega=[0,1]^{2}$ is investigated in this test. Let the diffusion tensors be

$$
\kappa_{\alpha}=\left\{\begin{array}{ll}
\left(\begin{array}{cc}
T_{\alpha} & 0 \\
0 & T_{\alpha}
\end{array}\right), & x \leq 0.5, \\
\left(\begin{array}{cc}
10 T_{\alpha} & 3 T_{\alpha} \\
3 T_{\alpha} & T_{\alpha}
\end{array}\right), & x>0.5,
\end{array} \quad \alpha=e, i, r .\right.
$$


Table 2: Numerical results of $T_{e}$ on random quadrilateral meshes at $t=0.1$.

\begin{tabular}{||ccccccccc||}
\hline Mesh & numkw & ms & as & $E_{u}$ & $R_{u}$ & $E_{q}$ & $R_{q}$ & $\overline{\text { iter }}$ \\
\hline $8 \times 8$ & 147 & 11 & 9.37 & $1.29 \mathrm{e}-3$ & - & $2.85 \mathrm{e}-2$ & - & 6.75 \\
$16 \times 16$ & 675 & 11 & 10.22 & $4.07 \mathrm{e}-4$ & 1.83 & $1.77 \mathrm{e}-2$ & 0.75 & 7.92 \\
$32 \times 32$ & 2883 & 11 & 10.62 & $1.17 \mathrm{e}-4$ & 1.83 & $1.04 \mathrm{e}-2$ & 0.79 & 7.95 \\
$64 \times 64$ & 11907 & 11 & 10.81 & $3.22 \mathrm{e}-5$ & 1.95 & $5.32 \mathrm{e}-3$ & 1.01 & 8.00 \\
$128 \times 128$ & 48387 & 11 & 10.91 & $8.63 \mathrm{e}-6$ & 1.93 & $2.75 \mathrm{e}-3$ & 0.97 & 8.00 \\
\hline
\end{tabular}

Table 3: Numerical results of $T_{e}$ on Kershaw meshes at $t=0.1$.

\begin{tabular}{||ccccccccc||}
\hline Mesh & numkw & $\mathrm{ms}$ & as & $E_{u}$ & $R_{u}$ & $E_{q}$ & $R_{q}$ & $\overline{\text { iter }}$ \\
\hline $8 \times 8$ & 147 & 11 & 9.37 & $2.34 \mathrm{e}-3$ & - & $4.87 \mathrm{e}-2$ & - & 10.00 \\
$16 \times 16$ & 675 & 11 & 10.22 & $8.65 \mathrm{e}-4$ & 1.70 & $2.65 \mathrm{e}-2$ & 1.03 & 17.00 \\
$32 \times 32$ & 2883 & 11 & 10.62 & $2.88 \mathrm{e}-4$ & 1.70 & $1.02 \mathrm{e}-2$ & 1.48 & 14.71 \\
$64 \times 64$ & 11907 & 11 & 10.81 & $8.44 \mathrm{e}-5$ & 1.83 & $3.19 \mathrm{e}-3$ & 1.74 & 12.88 \\
$128 \times 128$ & 48387 & 11 & 10.91 & $2.41 \mathrm{e}-5$ & 1.84 & $1.46 \mathrm{e}-3$ & 1.15 & 10.00 \\
\hline
\end{tabular}

Table 4: Numerical results of $T_{e}$ on polygonal meshes at $t=0.1$.

\begin{tabular}{||ccccccccc||}
\hline Mesh level & numkw & $\mathrm{ms}$ & as & $E_{u}$ & $R_{u}$ & $E_{q}$ & $R_{q}$ & $\overline{\text { iter }}$ \\
\hline 1 & 384 & 17 & 13.39 & $1.70 \mathrm{e}-3$ & - & $3.71 \mathrm{e}-2$ & - & 6.00 \\
2 & 1536 & 17 & 14.07 & $5.78 \mathrm{e}-4$ & 1.64 & $1.62 \mathrm{e}-2$ & 1.25 & 6.94 \\
3 & 6144 & 17 & 14.54 & $1.58 \mathrm{e}-4$ & 1.90 & $5.78 \mathrm{e}-3$ & 1.51 & 6.99 \\
4 & 24576 & 17 & 14.77 & $4.28 \mathrm{e}-5$ & 1.89 & $2.27 \mathrm{e}-3$ & 1.35 & 6.00 \\
5 & 98304 & 17 & 14.89 & $1.11 \mathrm{e}-5$ & 1.95 & $8.50 \mathrm{e}-4$ & 1.42 & 6.70 \\
\hline
\end{tabular}

The exact solution is

$$
T_{\alpha}(x, y, t)=\left\{\begin{array}{ll}
1-2 y^{2}+4 x y+6 x+2 y+t, & x \leq 0.5, \\
-2 y^{2}+1.6 x y-0.6 x+3.2 y+4.3+t, & x>0.5,
\end{array} \quad \alpha=e, i, r .\right.
$$

Other parameters are chosen as follows:

$$
\left\{\begin{array}{l}
\rho=1, \\
\omega_{e \alpha}=T_{e}, \quad \alpha=e, i, \\
c_{v \alpha}=100, \quad \alpha=e, i, \quad c_{v r}=25 / T_{r}^{3} .
\end{array}\right.
$$

This problem is the extension of the steady test inspired in [20]. Here we investigate the convergence on two mesh types, that is Mesh1 and Mesh2 where all the mesh nodes located on the line $x=0.5$ are distorted only in the $y$-direction. The final time and the time interval are the same as that in the previous example.

Numerical errors of the test are graphically depicted in Fig. 4 as log-log plots of the discrete errors versus the characteristic mesh size $h$. It implies that the scheme achieves second-order (resp. first-order) for the $L^{2}$ (resp. $H^{1}$ ) error for the discontinuous problem. 

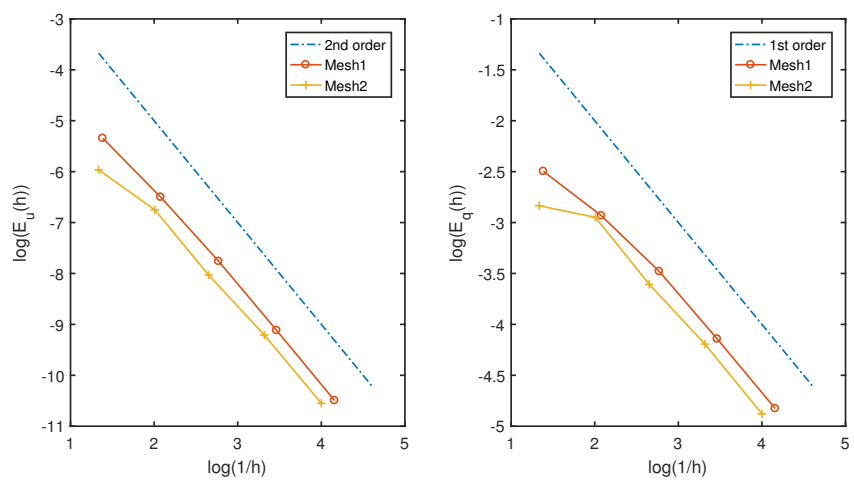

Figure 4: $L^{2}$ and $H^{1}$ errors of $T_{e}$ on Mesh1-Mesh2 at $t=0.1$.

\subsection{Numerical simulation}

In this subsection, we simulate some radiation diffusion problems which are not very far from the problems encountered in the context of the numerical simulation of Inertial Confinement Fusion. Denote the vertex-centered and positivity-preserving scheme in this paper as VPPS. The first scheme suggested in [51] (resp. [7]) for twodimensional two-temperature (resp. two-dimensional three-temperature) radiation diffusion problems is denoted as FVEM-2T (resp. FVEM-3T). In this subsection, FVEM-2T and FVEM-3T are also implemented and the corresponding results are presented whenever there is a need for comparison.

\subsubsection{A nonlinear thermal wave}

Consider a nonlinear thermal wave investigated in [25] which is the solution of the following nonlinear diffusion equation

$$
\rho C \frac{d T}{d t}-\nabla \cdot(D(T) \nabla T)=0,
$$

where the mass density and the heat capacity are given by $\rho=1$ and $C=1$. The nonlinear diffusion coefficient is given by $D(T)=T^{5 / 2}$. This type of nonlinearity corresponds to the so-called Spitzer-Härm conductivity which is frequently encountered in plasma physics for electron energy transport, see [49]. The problem is solved on a cylindrical domain $\Omega$ characterized by $r \in[0,1]$ and $\theta \in[0, \pi / 2]$ knowing that $r=\sqrt{x^{2}+y^{2}}$ and $\theta=\arctan \frac{y}{x}$, where $(x, y)$ denotes the Cartesian coordinates of a generic point inside the domain. The initial condition is given by $T(\boldsymbol{x}, 0)=1$. There are homogeneous flux boundary conditions along axis $x=0$ and $y=0$ and the normal flux is 1000 at the outer radius $r=1$. The unsteady solution is computed until time $t=0.003$. At this time a nonlinear heat wave has propagated into the cold medium. This wave is characterized by a sharp transition zone displaying a strong temperature gradient. Due to the boundary conditions and the geometry of the domain, the solution of the diffusion equation exhibits a cylindrical symmetry, namely $T(\boldsymbol{x})=T(r)$. 


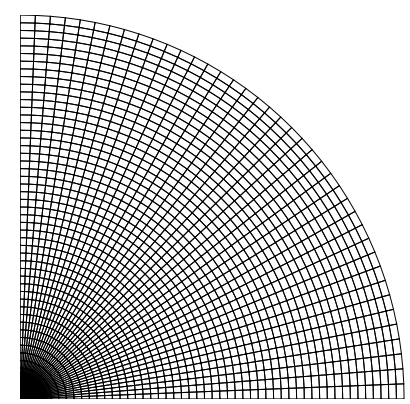

(a) $50 \times 40$ regular polar mesh

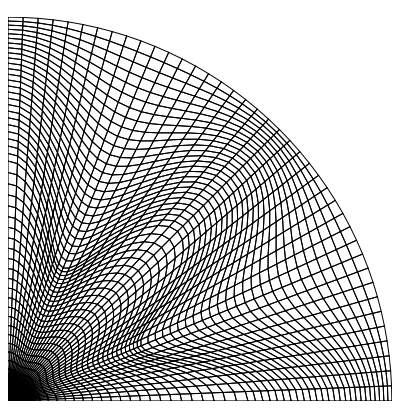

(b) $50 \times 40$ distorted polar mesh

Figure 5: Polar meshes for the nonlinear thermal wave problem.

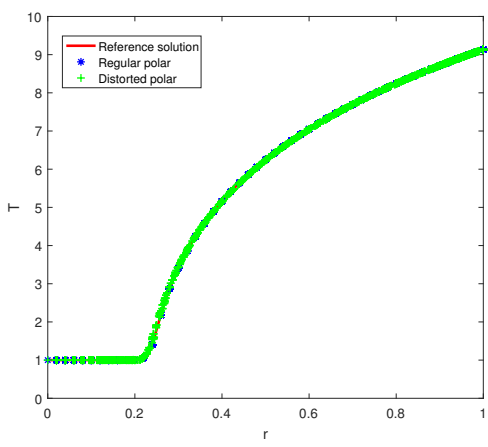

(a) Whole domain

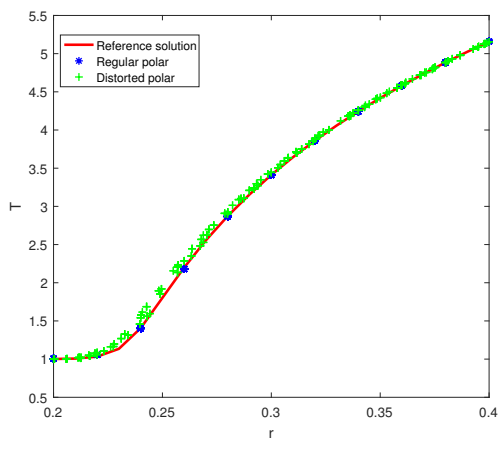

(b) Zoom in the vicinity of the thermal front

Figure 6: Numerical temperatures at the stopping time $t=0.003$.

As shown in Fig. 5, here we construct $50 \times 40$ regular and distorted polar meshes designed in [25]. Since this problem does not admit exact analytic solution, we compute the reference numerical solution using a $100 \times 90$ regular polar mesh. Then the numerical temperatures computed by the scheme on the meshes in Fig. 5 in all vertices as function of the vertex radius versus the reference solution are displayed in Fig. 6, which is similar to the results in [25]. The scheme bring us quite good results since they match very well to the reference solution and almost preserve the cylindrical symmetry of the solution.

\subsubsection{Two-temperature radiation diffusion problem}

Consider the nonlinear system of equations for non-equilibrium diffusion coupled to material conduction 


$$
\begin{aligned}
& \frac{\partial E}{\partial t}-\nabla \cdot(D \nabla E)=\sigma_{a}\left(T^{4}-E\right) \\
& \frac{\partial T}{\partial t}-\nabla \cdot(\kappa \nabla T)=\sigma_{a}\left(E-T^{4}\right)
\end{aligned}
$$

where $E$ is the radiation energy density, $D$ is the radiation diffusion coefficient, $\sigma_{a}$ is the photon absorption cross section, $T$ is the material temperature and $\kappa$ is the material conduction coefficient, respectively.

The energy exchange is controlled by the photon absorption cross section $\sigma_{a}$, which is modeled by

$$
\sigma_{a}=\frac{z^{3}}{T^{3}}
$$

where the atomic number $z=z(x, y)$ is a material coefficient, a function of position $(x, y)$. The radiation diffusion coefficient without flux limiter is

$$
D=\frac{1}{3 \sigma_{a}} .
$$

The radiation diffusion coefficient with flux limiter is

$$
D=\frac{1}{3 \sigma_{a}+\frac{|\nabla E|}{E}}
$$

The material conduction coefficient $\kappa$ has the following form

$$
\kappa=c_{0} T^{\frac{5}{2}}
$$

where $c_{0}=0.01$.

In this test, we solve the problem (5.1a)-(5.1b) on $\Omega=[0,1]^{2}$. The value of $z$ is

$$
z= \begin{cases}10, & \left(\frac{3}{16}, \frac{7}{16}\right) \times\left(\frac{9}{16}, \frac{13}{16}\right) \cup\left(\frac{9}{16}, \frac{13}{16}\right) \times\left(\frac{3}{16}, \frac{7}{16}\right), \\ 1, & \text { otherwise. }\end{cases}
$$

All four walls are insulated with respect to radiation diffusion and material conduction, i.e.,

$$
\left.\frac{\partial E}{\partial x}\right|_{x=0}=\left.\frac{\partial E}{\partial x}\right|_{x=1}=\left.\frac{\partial E}{\partial y}\right|_{y=0}=\left.\frac{\partial E}{\partial y}\right|_{y=0}=0,
$$

and

$$
\left.\frac{\partial T}{\partial x}\right|_{x=0}=\left.\frac{\partial T}{\partial x}\right|_{x=1}=\left.\frac{\partial T}{\partial y}\right|_{y=0}=\left.\frac{\partial T}{\partial y}\right|_{y=0}=0 .
$$

The initial condition is presented by

$$
E(r)=0.001+100 e^{-100 r^{2}}, \quad T(r)=E(r)^{1 / 4},
$$

where $r=\sqrt{x^{2}+y^{2}}$ is the distance from the lower left corner. The initial values of $T$ is shown in Fig. 7. Uniformly rectangular, randomly quadrilateral and triangular 


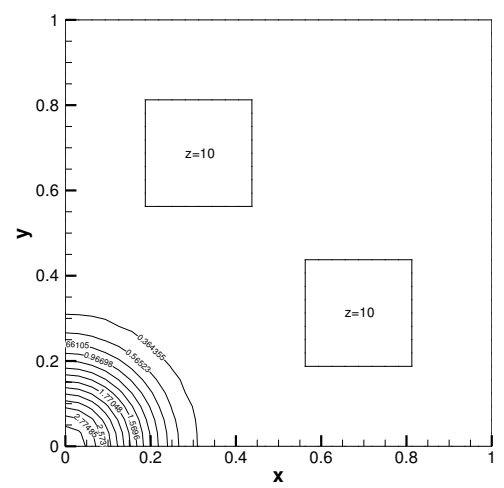

Figure 7: Initial conditions.
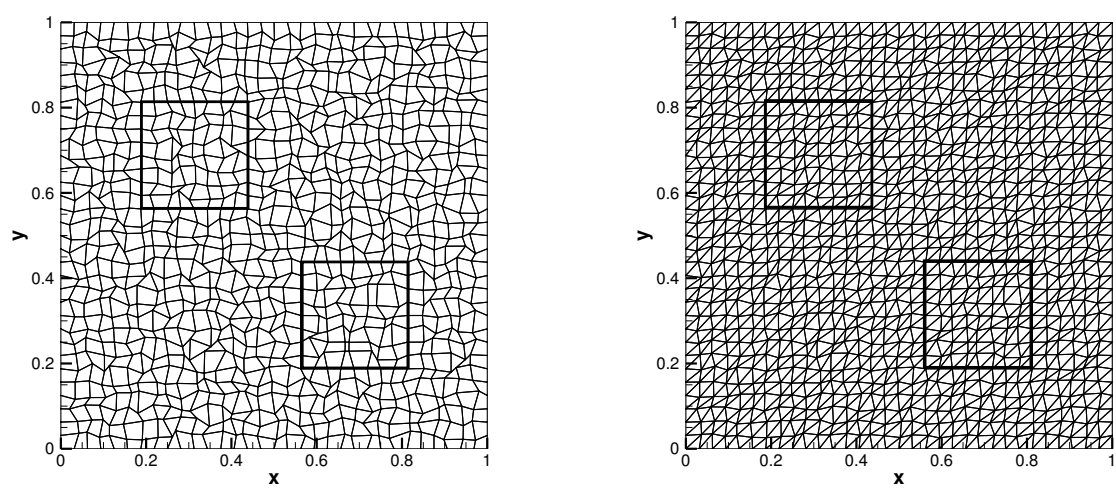

Figure 8: Randomly quadrilateral mesh (left) and randomly triangular mesh (right).

meshes are used in this test, which are shown in Fig. 8. Since the edges of these obstacles correspond exactly to the edges of primary meshes, there is no mixed material primary mesh. Here we emphasize that the present scheme can be used to solve two dimensional two-temperature radiation diffusion equations correspondingly.

In the following computations, we will use uniformly rectangular and randomly quadrilateral meshes with $96 \times 96$ cells and randomly triangular mesh with $96 \times 96 \times 2$ cells. In addition, we take the time interval $\tau=5 \times 10^{-4}$ throughout this test. Define the energy conservative error at the $n$-th time step by

$$
\text { Error }=\left|\mathcal{E}^{n}-\mathcal{E}^{0}\right|
$$

where

$$
\mathcal{E}^{0}=\sum_{\boldsymbol{x}_{\nu} \in \bar{\Omega}}\left(E_{\nu}^{0}+T_{\nu}^{0}\right)\left|K_{\nu}^{*}\right|
$$



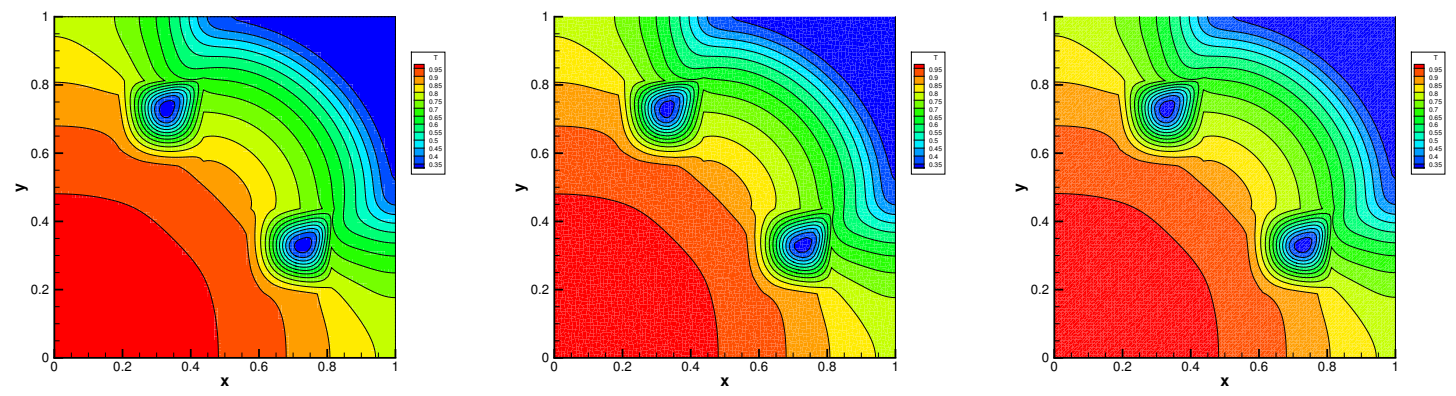

Figure 9: The radiation temperature at $t=1.5$ for the problem without flux limiter on uniformly rectangular mesh, randomly quadrilateral and triangular meshes.

Table 5: Numerical results of VPPS for the problem without flux limiter.

\begin{tabular}{||lcccccc||}
\hline Meshes & numkw & umin & umax & $L^{2}$-norm & $\overline{\text { iter }}^{\text {iter }}$ & $\overline{\text { A }}^{\text {A }}$ \\
Uniformly rectangular & 18818 & $1.00 \mathrm{E}-2$ & 0.9880 & 1.27068 & 25.61 & 9.08 \\
Randomly quadrilateral & 18818 & $1.00 \mathrm{E}-2$ & 0.9879 & 1.27059 & 29.48 & 9.85 \\
Randomly triangular & 18818 & $1.00 \mathrm{E}-2$ & 0.9881 & 1.27146 & 26.66 & 9.50 \\
\hline
\end{tabular}

is the total energy at the initial time and

$$
\mathcal{E}^{n}=\sum_{\boldsymbol{x}_{\nu} \in \bar{\Omega}}\left(E_{\nu}^{n}+T_{\nu}^{n}\right)\left|K_{\nu}^{*}\right|
$$

is the total energy at the time $t_{n}$.

Results without flux limiter. In this part, let the final state be $t=1.5$. The radiation diffusion coefficient is defined in (5.2).

The contours of the radiation temperature on uniformly rectangular mesh, randomly quadrilateral and triangular meshes at the final state $t=1.5$ are shown in Fig. 9. Comparing these three figures, it is hard to find any visible difference. Moreover, numerical results for the problem without flux limiter are presented in Table 5. We find that the numerical solution is positive on the domain in three mesh types. The $L^{2}$-norm of the solution on randomly quadrilateral and triangular meshes closely approximates that on rectangular mesh. These results show that our positivity-preserving scheme obtains the correct numerical solution on random quadrilateral and triangular meshes. The energy error for the problem without flux limiter on these three mesh types are shown in Fig. 10, which implies the scheme is conservative.

In addition, numerical results of FVEM-2T for the problem without flux limiter are shown in Table 6 . We find that the $L^{2}$-norm of the numerical solution by FVEM-2T is a bit larger than that of VPPS on uniformly rectangular and randomly quadrilateral meshes. Comparing the values of $\overline{i t e r}$ and $\overline{i t e r}^{A}$ in Table 5 and Table 6 , we find that Anderson Acceleration method reduces the number of iterations by nearly thrice.

Results with flux limiter. For the radiation diffusion coefficient with flux limiter in 


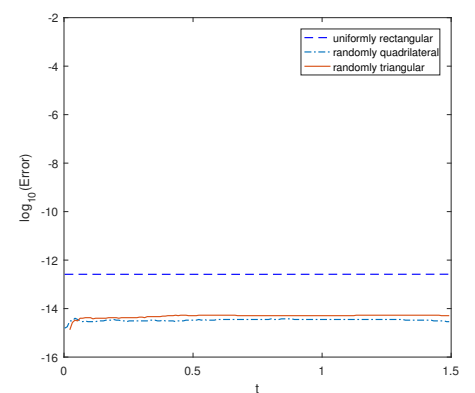

Figure 10: Energy error for the problem without flux limiter.

Table 6: Numerical results of FVEM-2T [51] for the problem without flux limiter.

\begin{tabular}{||lccc||}
\hline Meshes & $L^{2}$-norm & $\overline{\text { iter }}$ & $\overline{\text { iter }}^{A}$ \\
\hline Uniformly rectangular & 1.27087 & 25.62 & 9.08 \\
Randomly quadrilateral & 1.27077 & 28.53 & 9.10 \\
Randomly triangular & 1.27146 & 26.56 & 9.09 \\
\hline
\end{tabular}

(5.3), we choose

$$
D_{K}=\frac{1}{3 \frac{z_{K}^{3}}{T_{K}^{3}}+\frac{\left|\nabla E_{K}\right|}{E_{K}}},
$$

where $\nabla E_{K}$ is a discrete approximation to the gradient of $E$ on cell $K$. Here we present a computational formula to obtain $\nabla E_{K}$. By the Green formula and trapezoidal rule, we have

$$
\int_{K} \nabla E=\sum_{e \in \partial K} \int_{e} E \boldsymbol{n}_{e} d s \approx \sum_{e \in \partial K} \frac{1}{2}|e|\left(E_{\nu}+E_{\nu^{\prime}}\right) \boldsymbol{n}_{e},
$$

where $E_{\nu}$ and $E_{\nu^{\prime}}$ are two discrete approximations at the endpoints of $e, \boldsymbol{n}_{e}$ is the unit outward normal to $e$. Hence, we have

$$
\nabla E_{K}=\sum_{e \in \partial K} \frac{|e|}{2|K|}\left(E_{\nu}+E_{\nu^{\prime}}\right) \boldsymbol{n}_{e}
$$

In addition, we take the time interval $\tau=5 \times 10^{-4}$ and let the final state be $t=3.0$.

Fig. 11 presents the contours of the radiation temperature on various meshes at the final state $t=3.0$. One can see that the contours of the radiation temperature on randomly quadrilateral and triangular meshes accord with that on uniformly rectangular mesh. Table 7 lists the $L^{2}$-norms of the solution and number of iterations. From this table, we find that the $L^{2}$-norms of the solution on different meshes are similar, which implies the robustness of the scheme on distorted meshes. Moreover, the $L^{2}$-norms have small differences between VPPS and FVEM-2T. The values of $\overline{i t e r}$ and $\overline{i t e r}^{A}$ show the efficiency of Anderson acceleration algorithm. Energy errors on these three mesh 

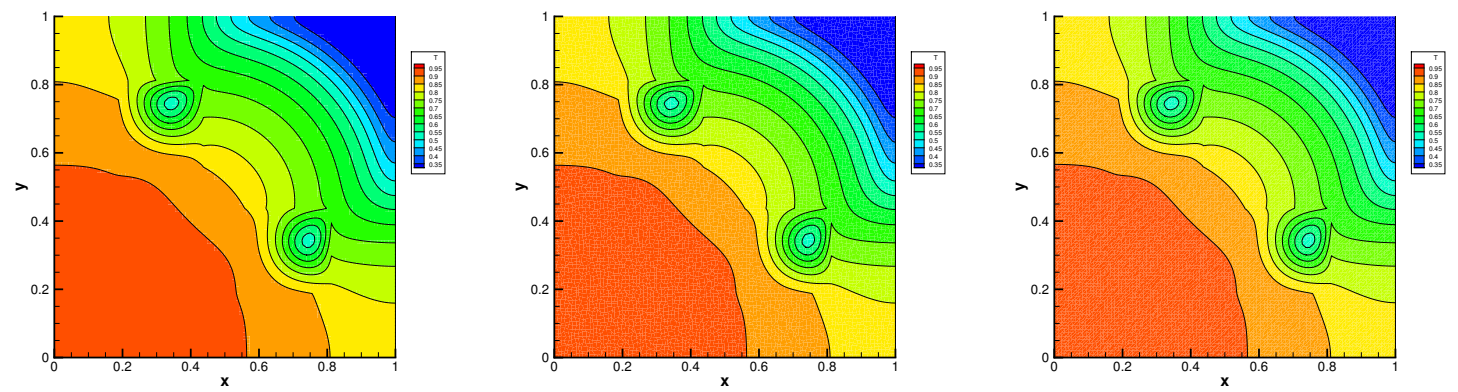

Figure 11: The radiation temperature at $t=3.0$ for the problem with flux limiter on uniformly rectangular, randomly quadrilateral and triangular meshes.

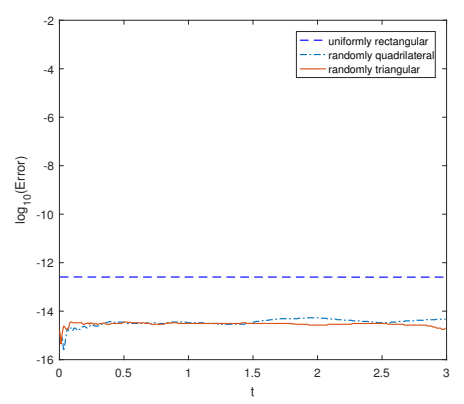

Figure 12: Energy error for the problem with flux limiter.

Table 7: Numerical results for the problem with flux limiter.

\begin{tabular}{||l|ccc|ccc||}
\hline \multirow{2}{*}{ Meshes } & \multicolumn{3}{|c|}{ VPPS } & \multicolumn{3}{c||}{ FVEM-2T [51] } \\
\cline { 2 - 7 } & $L^{2}$-norm & $\overline{\text { iter }}$ & $\overline{\text { iter }}^{A}$ & $L^{2}$-norm & $\overline{\text { iter }}$ & $\overline{\text { iter }}^{A}$ \\
\hline Uniformly rectangular & 1.23852 & 24.73 & 9.62 & 1.23877 & 24.78 & 9.65 \\
Randomly quadrilateral & 1.23850 & 26.12 & 9.98 & 1.23871 & 25.78 & 9.82 \\
Randomly triangular & 1.23953 & 25.26 & 9.99 & 1.23953 & 25.05 & 9.73 \\
\hline
\end{tabular}

types are described in Fig. 12. We find that the conservation errors are very small, which verifies that our scheme is conservative.

\subsubsection{Three-temperature radiation diffusion problem}

The typical model of laser-driven implosion of Inertial Confinement Fusion in [27] was taken as a benchmark. As shown in Fig. 13, the computation region $\Omega$ with boundary $\partial \Omega=\Gamma_{1} \cup \Gamma_{2}$ is a half circle with radius $R$ on the 2-D Cartesian coordinate plane. The circle center is at the coordinate origin and the diameter is overlapped with the $x$-axis. It is divided into three subregions. The innermost, middle and outside subdomains cover with $0<r<R_{1}, R_{1}<r<R_{2}$ and $R_{2}<r<R_{3}$ and are denoted as $\Omega_{i}$, $i=1,2,3$, respectively. 


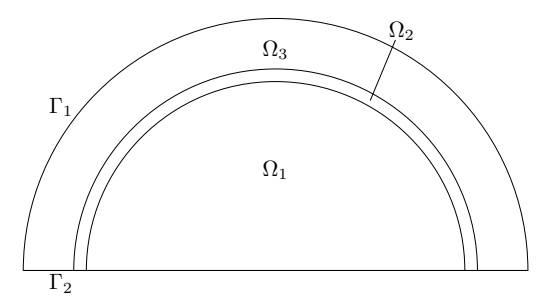

Figure 13: Computation domain.

The three subregions in Fig. 13 are filled with deuterium gas (DT), glass $\left(\mathrm{SiO}_{2}\right)$ and plastic foam $(\mathrm{CH})$, respectively. The values of all parameters are shown as follows:

$$
\begin{aligned}
& \left\{\begin{array}{l}
R_{1}=90, \\
R_{2}=95, \\
R_{3}=132,
\end{array} \quad \rho=\left\{\begin{array} { l } 
{ 0 . 0 9 \text { in } \Omega _ { 1 } , } \\
{ 2 . 5 0 \text { in } \Omega _ { 2 } , } \\
{ 1 . 1 0 \text { in } \Omega _ { 3 } , }
\end{array} \quad \left\{\begin{array}{l}
c_{v e}=1.5 \Gamma_{e}, \\
c_{v i}=1.5 \Gamma_{i}, \\
c_{v r}=\Gamma_{r}
\end{array}\right.\right.\right. \\
& \Gamma_{e}=\left\{\begin{array}{l}
35 \text { in } \Omega_{1}, \\
40 \text { in } \Omega_{2}, \\
45 \text { in } \Omega_{3},
\end{array} \quad \Gamma_{i}=\left\{\begin{array}{l}
35 \text { in } \Omega_{1}, \\
40 \text { in } \Omega_{2}, \\
70 \text { in } \Omega_{3},
\end{array} \quad \Gamma_{r}=0.007568,\right.\right. \\
& A_{e}=\left\{\begin{array}{ll}
200 & \text { in } \Omega_{1}, \\
60 & \text { in } \Omega_{2}, \\
81 & \text { in } \Omega_{3},
\end{array} \quad A_{i}=\left\{\begin{array}{ll}
5 & \text { in } \Omega_{1}, \\
1.7 \times 10^{-7} & \text { in } \Omega_{2}, \\
2.0 \times 10^{-2} & \text { in } \Omega_{3},
\end{array} \quad A_{r}= \begin{cases}1.8 \times 10^{7} / \rho & \text { in } \Omega_{1}, \\
9.0 \times 10^{2} / \rho^{1.5} & \text { in } \Omega_{2}, \\
2.1 \times 10^{3} / \rho^{2} & \text { in } \Omega_{3},\end{cases} \right.\right. \\
& \beta=\left\{\begin{array}{ll}
1.0 & \text { in } \Omega_{1}, \\
2.4 & \text { in } \Omega_{2}, \\
3.0 & \text { in } \Omega_{3},
\end{array} \quad A_{e i}=\left\{\begin{array}{l}
2000 \text { in } \Omega_{1}, \\
4000 \text { in } \Omega_{2}, \\
7000 \text { in } \Omega_{3},
\end{array} \quad A_{e r}= \begin{cases}10 & \text { in } \Omega_{1}, \\
140 & \text { in } \Omega_{2}, \\
79 & \text { in } \Omega_{3} .\end{cases} \right.\right.
\end{aligned}
$$

In this test, let $\Gamma_{1}$ and $\Gamma_{2}$ be the free surfaces and rigid walls. The boundary and initial-value conditions are introduced in (2.2) and (2.3), respectively. Meanwhile, the boundary value and the initial value are

$$
g_{D}(\boldsymbol{x}, t)=2
$$

and

$$
g_{\alpha}(\boldsymbol{x})=3 \times 10^{-4}, \quad \alpha=e, i, r,
$$

respectively. Obviously, this radiation diffusion problem is a nonlinear heat wave problem.

The time interval is taken as $\tau=5 \times 10^{-4}$. Fig. 14 presents four mesh types, to be used in this tests. They are unstructured triangular mesh, quadrilateral mesh, polygonal mesh and non-matching mesh. Here we remark that the scheme FVEM-3T can only work on unstructured triangular mesh.

In the numerical simulation, we use the following formula to compute the relative energy conservative error

$$
\text { Error }=\frac{\mathcal{E}_{\text {enter }}^{n}-\left(\mathcal{E}_{\text {own }}^{n}-\mathcal{E}_{\text {own }}^{0}\right)}{\mathcal{E}_{\text {enter }}^{n}+\mathcal{E}_{\text {own }}^{0}},
$$




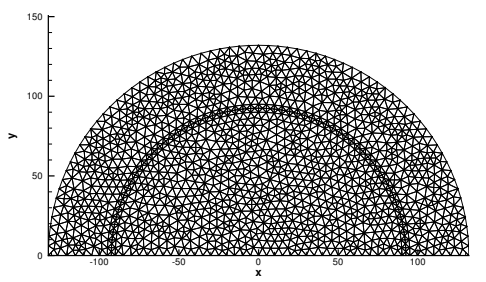

(a) Unstructured triangular mesh

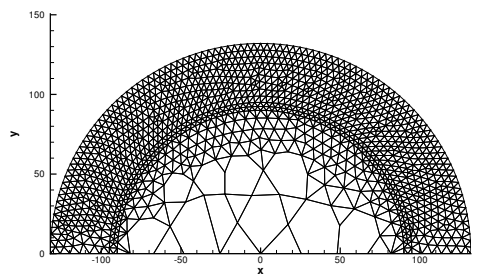

(c) Polygonal mesh

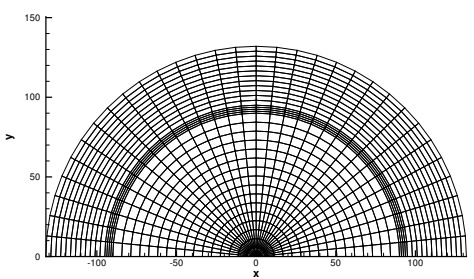

(b) Quadrilateral mesh

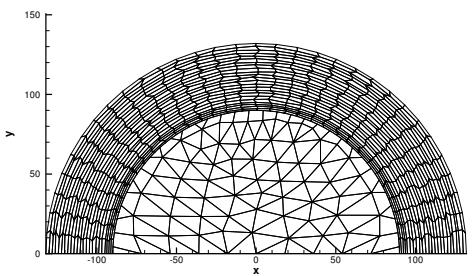

(d) Non-matching mesh

Figure 14: Four mesh types.

where $\mathcal{E}_{\text {enter }}^{n}$ denotes the total radiation energy importing from Dirichlet boundary, $\mathcal{E}_{\text {own }}^{n}$ and $\mathcal{E}_{\text {own }}^{0}$ computed by the formula (2.4) denote the system energy at time $t_{n}$ and $t_{0}=0$, respectively. Note that the computation of $\mathcal{E}_{\text {enter }}^{n}$ should define the discrete flux across the Dirichlet boundary. Here we first reconstruct the value of $\nabla T_{\alpha}$ by (5.4). Assume that $\sigma \in \partial K \cap \Gamma_{D}$, Then we results in the discrete flux across $\sigma$ by

$$
-\int_{\sigma} \kappa_{\alpha, K} \nabla T_{\alpha} \cdot \boldsymbol{n}_{\sigma} \approx-|\sigma| \kappa_{\alpha, K} \nabla T_{\alpha} \cdot \boldsymbol{n}_{K, \sigma},
$$

where $\boldsymbol{n}_{K, \sigma}$ denotes the outward unit vector normal to the boundary.

The temperature distributions of electron, ion and photon are presented from Figs. 15 to 18 at the specified time, $t=0.5,5.0$ and 50 respectively on four mesh types. Unlike the schemes based on the harmonic averaging of cell-centered diffusion coefficients, such as the cell-centered schemes in [9], the evolution of radiation energy and the exchange of energy among electrons, ions and photons are appropriate and comparable. Hence, we believe the present scheme effectively alleviates the numerical heat-barrier issue in this test. The temperatures of electron, ion and photon are positive and not exceeding 2 at each specified time. Moreover, the temperature of photon is higher than those of electron and ion at each specified time and the temperature distributions are all symmetric about the origin. It verifies the validity of the vertex-centered positivity-preserving scheme for the 2-D 3-T radiation diffusion problem.

Energy conservative error of schemes VPPS and FVEM-3T at various specified times are presented in Table 8 . We find that those two schemes possess the similar results.

Since the 2-D 3-T radiation diffusion problem is relatively complex, it is extremely difficult to theoretically analyze the convergent accuracy of the scheme. Therefore, we 


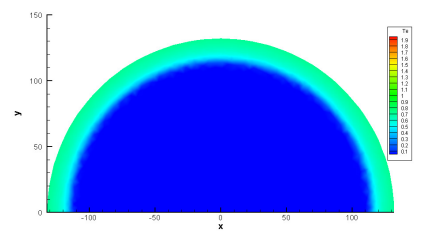

(a) Electron

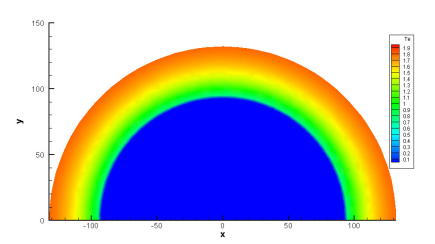

(d) Electron

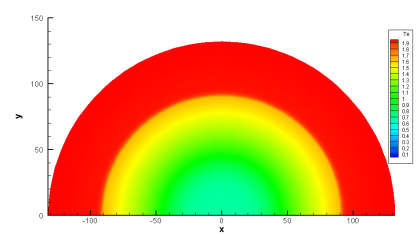

(g) Electron

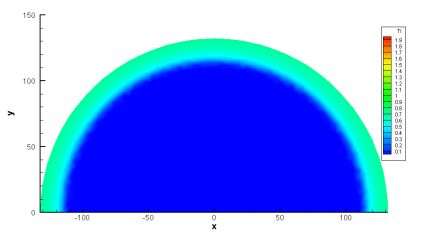

(b) Ion

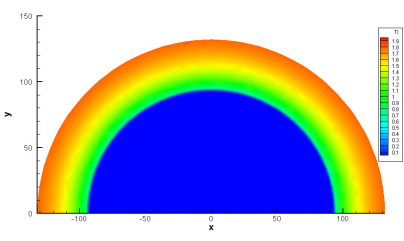

(e) Ion

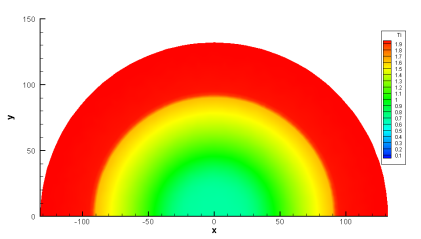

(h) Ion

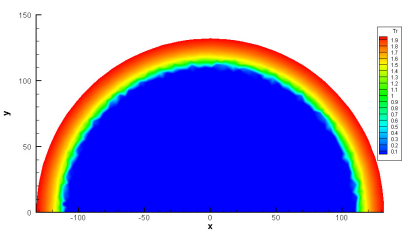

(c) Photon

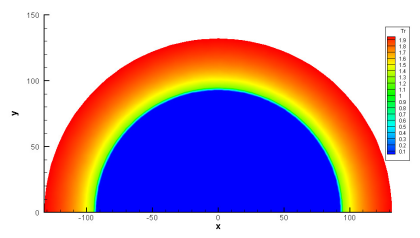

(f) Photon

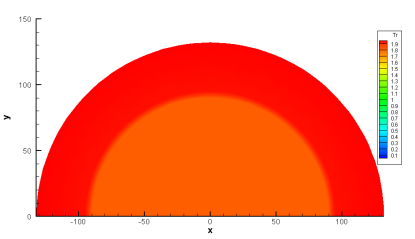

(i) Photon

Figure 15: Numerical solutions on unstructured triangular mesh at $T=0.5,5.0,50.0$.

Table 8: Energy conservative error (\%) on unstructured triangular mesh.

\begin{tabular}{||c|cccccc||}
\hline \multirow{2}{*}{ Scheme } & \multicolumn{5}{|c||}{ Physical time } \\
\cline { 2 - 7 } & 0.1 & 1.0 & 5.0 & 10.0 & 25.0 & 50.0 \\
\hline VPPS & 10.909247 & 5.518378 & 2.108630 & 1.294443 & 0.757553 & 0.240218 \\
FVEM-3T [7] & 10.909249 & 5.518375 & 2.102154 & 1.284038 & 0.693114 & 0.234765 \\
\hline
\end{tabular}

Table 9: Energy conservative error (\%) on quadrilateral mesh with three levels.

\begin{tabular}{||c|cccccccc||}
\hline \multirow{2}{*}{ Mesh level } & \multicolumn{8}{|c||}{ Physical time } \\
\cline { 2 - 9 } & 0.1 & 1.0 & 5.0 & 10.0 & 20.0 & 30.0 & 40.0 & 50.0 \\
\hline 1 & 16.80 & 9.31 & 5.08 & 3.94 & 3.30 & 2.93 & 2.68 & 2.50 \\
2 & 7.30 & 4.32 & 2.45 & 1.92 & 1.61 & 1.45 & 1.33 & 1.25 \\
3 & 3.36 & 2.08 & 1.20 & 0.94 & 0.79 & 0.72 & 0.66 & 0.62 \\
\hline
\end{tabular}

can only discuss the approximation accuracy from the view of numerical test. Numerical results on energy conservative errors are shown in Table 9. One can see that the energy conservative error becomes nearly half as mesh grid is uniformly refined and that it is convergent of order one. This also confirms the correctness and robustness of the new scheme. 


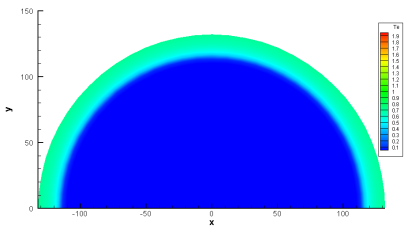

(a) Electron

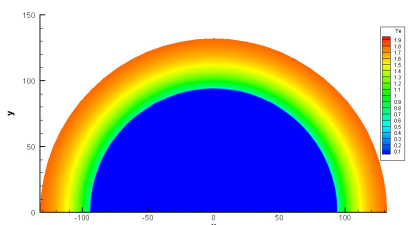

(d) Electron

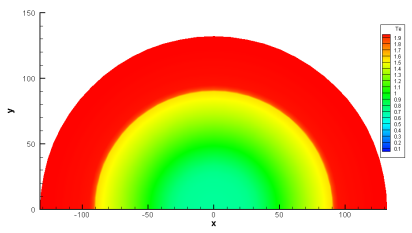

(g) Electron

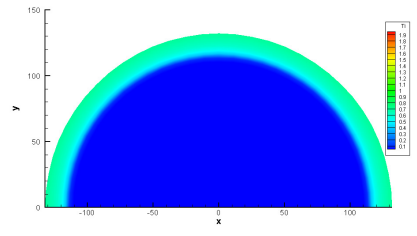

(b) Ion

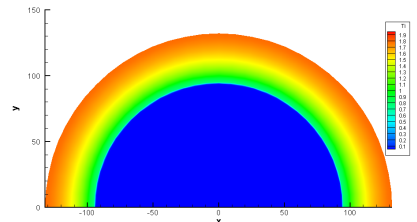

(e) Ion

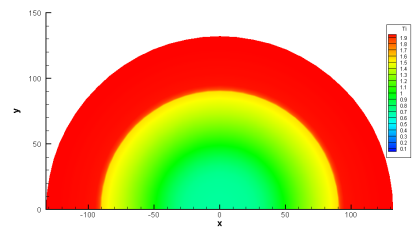

(h) Ion

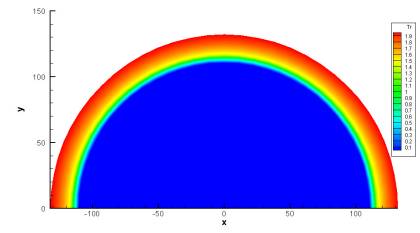

(c) Photon

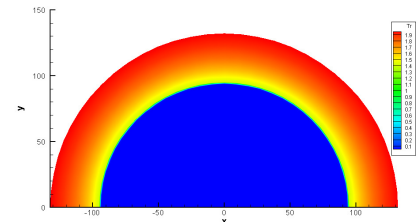

(f) Photon

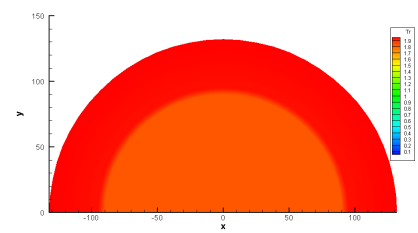

(i) Photon

Figure 16: Numerical solutions on quadrilateral mesh at $T=0.5,5.0,50.0$.

\section{Conclusions}

In this paper, a pure vertex-centered and positivity-preserving finite volume scheme is suggested for two-dimensional three-temperature radiation diffusion equations on general polygonal meshes with star-shaped cells. The scheme abandons the traditional convex decomposition of the co-normal vector and the auxiliary unknowns defined at cell-centers and edge midpoints are evaluated by a simple interpolation algorithm. Numerical results show that the present scheme achieves a second-order accuracy, generates nonnegative discrete solutions on arbitrary star-shaped polygonal meshes. The contours of numerical solution obtained by our scheme on various distorted meshes accord with that on smooth meshes for both two-temperature and three-temperature non-equilibrium radiation diffusion equations. Moreover, Anderson acceleration of the Picard method is applied to solve the resulting nonlinear system and demonstrates its less computational costs. These results show that our positivity-preserving scheme is a practical and attractive method for solving non-equilibrium radiation diffusion equations on polygonal meshes. Currently, some numerical codes have been proposed to simulate radiation hydrodynamics where the matter temperature is defined at the vertices, such as MULTI-2D in [34], TRHD in [38]. In the future, we will focus on the 


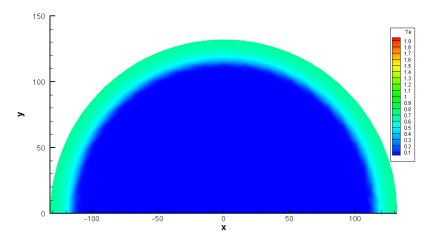

(a) Electron

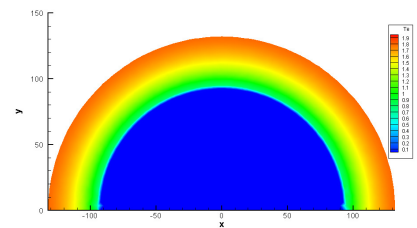

(d) Electron

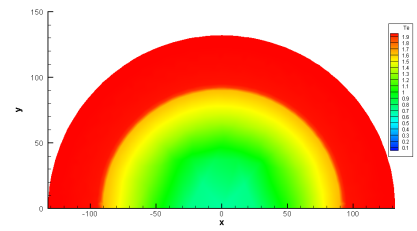

(g) Electron

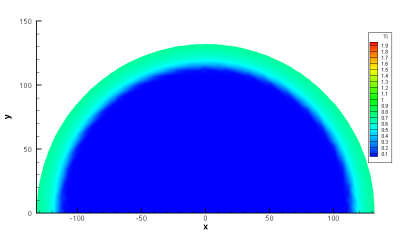

(b) Ion

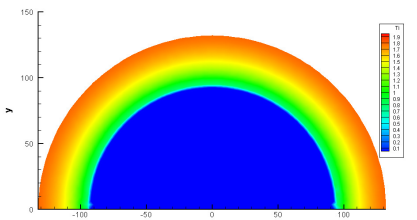

(e) Ion

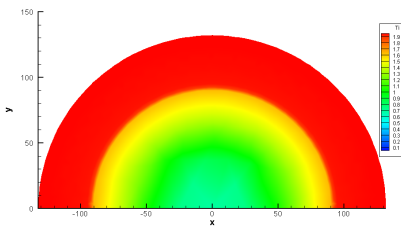

(h) Ion

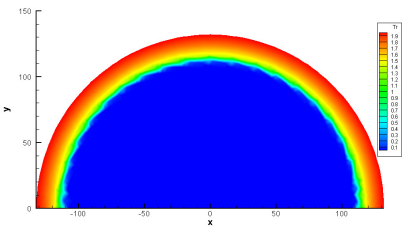

(c) Photon

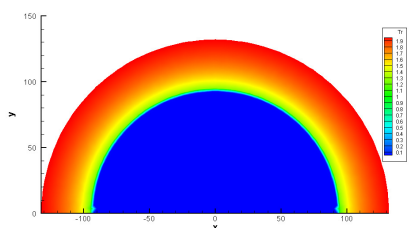

(f) Photon

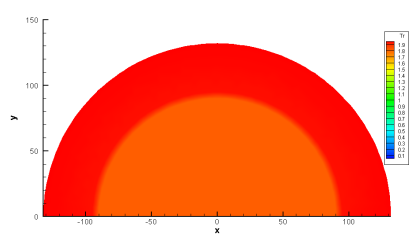

(i) Photon

Figure 17: Numerical solutions on polygonal mesh at $T=0.5,5.0,50.0$.

theoretical analysis and the extension of the present scheme to three temperature radiation hydrodynamics.

Acknowledgements The authors wish to thank Dr. Yanni Gao and Dr. Cunyun Nie for valuable discussions and for providing us some mesh data in Fig. 11. The authors also thank the anonymous reviewers for their careful reading and useful suggestions. This work was partially supported by the National Natural Science Foundation of China (No. 11871009) and Postdoctoral Research Foundation of China (No. BX20190013).

\section{References}

[1] H. An, X. JiA AND H. F. WALKer, Anderson acceleration and application to the threetemperature energy equations, J. Comput. Phys., 347 (2017), pp. 1-19.

[2] H. AN, Z. Mo, X. XU AND X. LIU, On choosing a nonlinear initial iterate for solving the 2-D 3-T heat conduction equations, J. Comput. Phys., 228(9) (2009), pp. 3268-3287.

[3] D. G. ANDERson, Iterative procedures for nonlinear integral equations, J. Assoc. Comput. Mach., 12(4) (1965), pp. 547-560. 


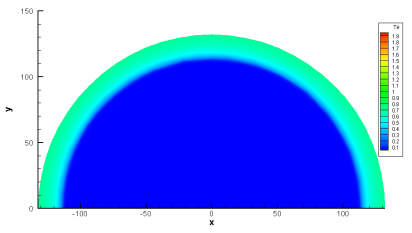

(a) Electron

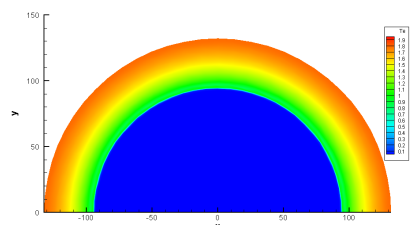

(d) Electron

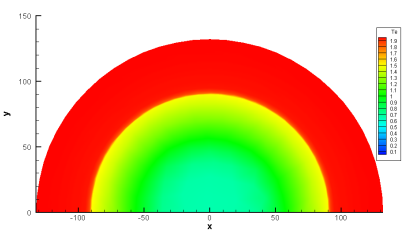

(g) Electron

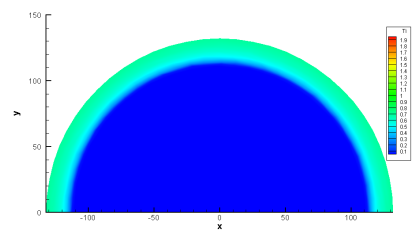

(b) Ion

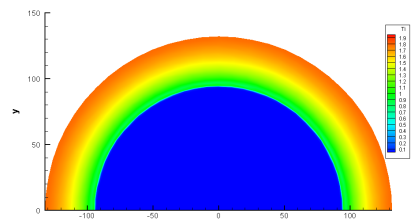

(e) Ion

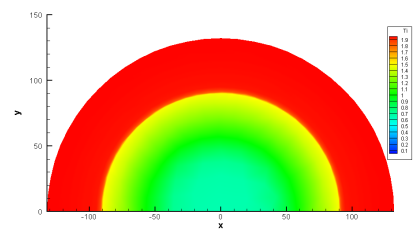

(h) Ion

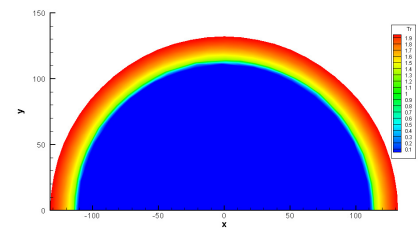

(c) Photon

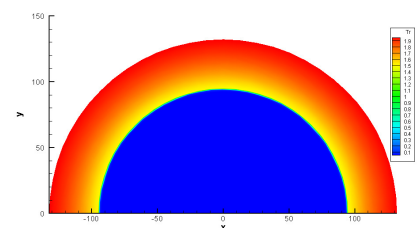

(f) Photon

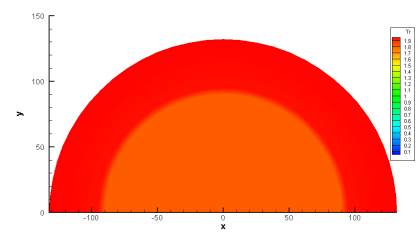

(i) Photon

Figure 18: Numerical solutions on non-matching mesh at $T=0.5,5.0,50.0$.

[4] W. W. DAI AND A. J. SCANNAPIECO, Interface- and discontinuity-aware numerical schemes for plasma 3-T radiation diffusion in two and three dimensions, J. Comput. Phys., 300 (2015), pp. 643-664.

[5] T. Feng, X. YU, H. AN, Q. Li AND R. Zhang, The preconditioned Jacobian-free NewtonKrylov methods for nonequilibrium radiation diffusion equations, J. Comput. Appl. Math., 255(4) (2014), pp. 60-73.

[6] S. FU, S. HuANG AND Y. LI, Numerical simulation of indirectly driven high convergence implosions, Chin. J. Comput. Phys., 16 (1999), pp. 162-166.

[7] Y. GAO, X. ZHAO AND Y. LI, Finite volume element methods for two-dimensional threetemperature radiation diffusion equations, Numer. Math. Theor. Meth. Appl., 9(3) (2016), pp. 470-496.

[8] Z. GAO AND J. WU, A linearity-preserving cell-centered scheme for the heterogeneous and anisotropic diffusion equations on general meshes, Int. J. Numer. Meth. Fluids, 67(12) (2011), pp. 2157-2183.

[9] Z. GAO AND J. WU, A second-order positivity-preserving finite volume scheme for diffusion equations on general meshes, SIAM. J. Sci. Comput., 37 (2015), pp. A420-A438.

[10] T. GU, Z. DAI AND X. LiU, Partial newton-krylov iterative methods for a system of energy equations with three-temperatures, Numer. Heat Transf. Part B Fundamentals, 53(3) (2008), pp. 259-270.

[11] J. A. Harte, W. E. Alley, D. S. Bailey, J. L. Eddleman and G. B. Zimmerman, Lasnexa 2-d physics code for modeling ICF, UCRL-LR-105821-96-4, 6(4) (1996). 
[12] Z. HUANG AND Y. LI, Monotone finite point method for non-equilibrium radiation diffusion equations, BIT Numer. Math., 56(2) (2016), pp. 659-679.

[13] D. JiA, Z. SHENG AND G. YUAN, An extremum-preserving iterative procedure for the imperfect interface problem, Commun. Comput. Phys., 25 (2019), pp. 853-870.

[14] J. JiAng, Y. HuAng, S. Shu AND S. Zeng, Some new discretization and adaptation and multigrid methods for 2-D 3-T diffusion equations, J. Comput. Phys., 224(1) (2007), pp. $168-181$.

[15] K. S. KANG, $P_{1}$ nonconforming finite element multigrid method for radiation transport, SIAM J. Sci. Comput., 25 (2003), pp. 369-384.

[16] D. A. KNOlL, R. B. LOWRIE AND J. E. Morel, Numerical analysis and time integration errors for nonequilibrium radiation diffusion, J. Comput. Phys., 226 (2007), pp. 13321347.

[17] D. A. KNoll, W. J. Rider ANd G. L. Olson, An efficient nonlinear solution method for non-equilibrium radiation diffusion, J. Quant. Spectrosc. Radiat. Transfer, 63 (1999), pp. $15-29$.

[18] S. H. Langer, H. A. Scott, M. M. Marinak and O. L. Landen, Comparisons of line emission from 2- and 3-dimensional simulations of ICF capsules to experiments, J. Quant. Spectrosc. Radiat. Transfer, 81(1-4) (2003), pp. 275-286.

[19] K. Lipnikov, G. MANzini, J. D. Moulton AND M. Shashkov, The mimetic finite difference method for elliptic and parabolic problems with a staggered discretization of diffusion coefficient, J. Comput. Phys., 305(15) (2016), pp. 111-126.

[20] K. LIPNIKOV, D. SVYATSKIY AND Y. VASSILEVSKI, Interpolation-free monotone finite volume method for diffusion equations on polygonal meshes, J. Comput. Phys., 228(3) (2009), pp. 703-716.

[21] C. Lu, W. HuAng AND E. S. V. VLeCK, The cutoff method for the numerical computation of nonnegative solutions of parabolic PDEs with application to anisotropic diffusion and lubrication-type equations, J. Comput. Phys., 242 (2013), pp. 24-36.

[22] J. LV, G. YUAN AND J. YUE, Nonnegativity-preserving repair techniques for the finite element solutions of degenerate nonlinear parabolic problems, Numer. Math. Theor. Meth. Appl., 11 (2018), pp. 413-436.

[23] D. MAdDix, L. SAmpaio AND M. GerRitSen, Numerical artifacts in the discontinuous generalized porous medium equation: How to avoid spurious temporal oscillations, J. Comput. Phys., 368 (2018), pp. 277-298.

[24] D. MAddix, L. SAMPAiO AND M. Gerritsen, Numerical artifacts in the generalized porous medium equation: Why harmonic averaging itself is not to blame, J. Comput. Phys., 361 (2018), pp. 280-298.

[25] P.-H. MAIRE AND J. BREIL, A nominally second-order accurate finite volume cell-centered scheme for anisotropic diffusion on two-dimensional unstructured grids, J. Comput. Phys., 231 (2012), pp. 2259-2299.

[26] G. Manzini, K. Lipnikov, J. D. Moulton and M. Shashkov, Convergence analysis of the mimetic finite difference method for elliptic problems with staggered discretizations of diffusion coefficients, SIAM J. Numer. Anal., 55(6) (2017), pp. 2956-2981.

[27] Z. Mo, L. SHEN AND G. WitTum, Parallel adaptive multigrid algorithm for 2-d 3-t diffusion equations, Int. J. Comput. Math., 81(3) (2004), pp. 361-374.

[28] V. A. Mousseau AND D. A. KNoll, New physics-based preconditioning of implicit methods for nonequilibrium radiation diffusion, J. Comput. Phys., 190 (2003), pp. 42-51.

[29] V. A. MousseAu AND D. A. KNOlL, Temporal accuracy of the nonequilibrium radiation diffusion equations applied to two-dimensional multimaterial simulations, Nucl. Sci. Eng., 
A Vertex-Centered Positivity-Preserving Scheme for 2-D 3-T Equations

154(2) (2006), pp. 174-189.

[30] V. A. Mousseau, D. A. Knoll and W. J. Rider, Physics-based preconditioning and the Newton-Krylov method for non-equilibrium radiation diffusion, J. Comput. Phys., 160 (2000), pp. 743-765.

[31] C. NiE AND H. YU, A Raviart-Thomas mixed finite element scheme for the two-dimensional three-temperature heat conduction problems, Int. J. Numer. Meth. Eng., 111 (2017), pp. 983-1000.

[32] G. L. OsLON, Efficient solution of multi-dimensional flux-limited non-equilibrium radiation diffusion coupled to material conduction with second-order time discretization, J. Comput. Phys., 226 (2007), pp. 1181-1195.

[33] H. Peng, Radiation Transport and Radiation Hydrodynamics in Plasmas, National Defense Indusrtry Press, Beijing, 2008.

[34] R. Ramis, J. Meyer ter Vehn and J. Ramirez, MUlTi2D-a computer code for twodimensional radiation hydrodynamics, Comput. Phys. Commun., 180 (2009), pp. 977994.

[35] M. SChNeIder, B. Flemisch And R. Helmig, Monotone nonlinear finite-volume method for nonisothermal two-phase two-component flow in porous media, Int. J. Numer. Meth. Fluids, 84(6) (2017), pp. 352-381.

[36] M. Schneider, B. Flemisch, R. Helmig, K. Terekhov and H. Tchelepi, Monotone nonlinear finite-volume method for challenging grids, Comput. Geosci., 22(2) (2018), pp. 565-586.

[37] C. D. SIJOY AND S. CHATURVEDI, TRHD: Three-temperature radiation-hydrodynamics code with an implicit non-equilibrium radiation transport using a cell-centered monotonic finite volume scheme on unstructured-grids, Comput. Phys. Commun., 190 (2015), pp. 98-119.

[38] C. D. SiJOY AND S. ChatuRVEDi, Combining node-centered parallel radiation transport and higher-order multi-material cell-centered hydrodynamics methods in three-temperature radiation hydrodynamics code TRHD, Comput. Phys. Commun., 203 (2016), pp. 94-109.

[39] H. A. VAN DER VORST, Bi-CGSTAB: a fast and smoothly converging variant of Bi-CG for the solution of nonsymmetric linear systems, SIAM J. Sci. Stat. Comput., 13(2) (1992), pp. 631-644.

[40] R. S. VARGA, Matrix Iterative Analysis, Second Revised and Expanded Edition, Springer Berlin Heidelberg, 2000.

[41] S. WANG, G. YUAN, Y. Li AND Z. SHENG, Discrete maximum principle based on repair technique for diamond type scheme of diffusion problems, Int. J. Numer. Meth. Fluids, 70(9) (2012), pp. 1188-1205.

[42] J. WU, Vertex-centered linearity-preserving schemes for nonlinear parabolic problems on polygonal grids, J. Sci. Comput., 71 (2017), pp. 499-524.

[43] X. XU, Z. Mo AND H. AN, Algebraic two-level iterative method for 2-D 3-T radiation diffusion equations, Chin. J. Comput. Phys., 26(1) (2009), pp. 1-8.

[44] X. YANG, W. HUANG AND J. QIU, Moving mesh finite difference solution of non-equilibrium radiation diffusion equations, Numer. Algor., 80(2) (2019).

[45] L. YIN, J. WU AND Z. DAI, A lions domain decomposition algorithm for radiation diffusion equations on non-matching grids, Numer. Math. Theor. Meth. Appl., 8(4) (2015), pp. 530548.

[46] J. Yue AND G. YuAn, Picard-Newton iterative method with time step control for multimaterial nonequilibrium radiation diffusion problem, Commun. Comput. Phys., 10 (2011), pp. 844-866.

[47] X. Yue, S. Shu, J. WANG And Z. Zhou, Substructuring preconditioners with a simple 
coarse space for 2-D 3-T radiation diffusion equations, Commun. Comput. Phys., 23(2) (2018), pp. 540-560.

[48] X. Yue, S. Shu, W. Xu And Z. Zhou, An adaptive combined preconditioner with applications in radiation diffusion equations, Commun. Comput. Phys., 18(5) (2015), pp. 1313-1335.

[49] Ya. B. Zel'dovich And Yu. P. Raizer, Physics of Shock Waves and High-Temperature Hydrodynamic Phenomena, vol. II, Academic Press, 1967.

[50] X. ZhANG, S. SU AND J. WU, A vertex-centered and positivity-preserving scheme for anisotropic diffusion problems on arbitrary polygonal grids, J. Comput. Phys., 344 (2017), pp. 419-436.

[51] X. Zhao, Y. Chen, Y. Gao, C. YU AND Y. LI, Finite volume element methods for nonequilibrium radiation diffusion equations, Int. J. Numer. Meth. Fluids, 73 (2013), pp. 1059-1080. 